\title{
Finite Total Cross-Sections in Nonrelativistic Quantum Mechanics
}

\author{
V. Enss ${ }^{1, \star}$ and B. Simon ${ }^{2, \star \star}$ \\ ${ }^{1}$ Institute for Advanced Study, Princeton, New Jersey 08540, USA \\ 2 Departments of Mathematics and Physics, Princeton University, Princeton, New Jersey 08544, USA
}

\begin{abstract}
We present a simple geometric method for estimating total crosssections in two-body and more generally two cluster scattering. We discuss a variety of aspects of total cross-sections including large coupling constant behavior.
\end{abstract}

\section{Introduction}

The total scattering cross-section is one of the basic objects in a quantum scattering problem. Nevertheless, there has been relatively little rigorous study of it until recently and, even in the physics literature, there appears to be no discussion of some very basic questions. For example, let $\sigma(\mathbf{k} ; V)$ denote the total cross-section for scattering involving the pair $\left(-\frac{1}{2} \Delta,-\frac{1}{2} \Delta+V\right)$ at incident momentum k. Except [37], we know of no study of the large $g$ behavior of $\sigma(\mathbf{k} ; g V)$. Here we will study this question (or more precisely, the growth of $\left.\int_{a}^{b} \sigma(k \mathbf{e} ; g V) d k\right)$ and obtain a bound (see Sect. 4) by $C g^{(v-1) /(\alpha-1)}$ if $V \sim|x|^{-\alpha}$ at infinity in $v$ dimensions with

$$
\alpha>\frac{1}{2}(v+1) .
$$

[(1.1) is needed for $\sigma$ to be finite.] In some special cases, we will obtain a lower bound with the same power behavior (see Appendix 2).

A second question which we mention explicitly is the finiteness of the total cross-section (including scattering into charged clusters or more than two clusters) for Coulomb scattering with two cluster initial state with both clusters neutral. Again, we know of no previous results on this problem, although Combes has informed us that he and Tip [12] have obtained similar results with different

* On leave from Dept. of Physics, University of Bielefeld, Federal Republic of Germany; address after April 1, 1980 : Dept. of Mathematics, University of Bochum, D-4630 Bochum 1, Federal Republic of Germany

$\star \star \quad$ Research partially aupported by USNSF under Grant MCS-78-01885 
methods and we expect that the work of Amrein et al., discussed below, could be extended to handle these situations.

One reason for the small amount of literature on the subject of total crosssections is that it would appear that time independent methods treat the problem en passant. After all, the total cross-section at fixed incident energy but averaged over incident angle is just the Hilbert-Schmidt norm of the on-shell T-matrix which is simply described in terms of continuum eigenfunctions. In particular, Kuroda [34], as a by-product of his analysis of eigenfunction expansions, obtained sufficient conditions for the $T$-matrix to be in the trace ideal $\mathscr{I}_{p}$. His conditions cannot be much improved since there will be examples with only slightly less power falloff for which the Born term in the $T$-matrix is not in $\mathscr{I}_{p}$. In addition, Agmon [1] under suitable smoothness hypotheses on $V$ has proven continuity (and thus boundedness) of $T$ away from the forward direction and thus boundedness of the non-forward differential cross-section; Agmon only needs $|x|^{-1-\varepsilon}$ falloff on $V$ (together with greater falloff on derivatives).

These consequences of the time-independent theory are not the end of the story for two reasons: first there is no definitive time independent theory for general $\mathrm{N}$ body systems, especially systems with Coulomb potentials. Moreover, even in the two-body case, the time-independent theory is unsatisfactory in the sense that one solves the Lippman-Schwinger equation by appealing to a Fredholm alternative and for this reason, one has no control on the size of the solutions and of the $T$ matrix ; that is, one knows $\sigma_{\text {tot }}$ is finite on the basis of Kuroda's work but except in special regimes (large energy or small coupling where the Born series converges) one has no idea of how large $\sigma_{\text {tot }}$ can be. In particular, control on the growth for large coupling seems unlikely by conventional time-independent methods.

The Kato-Birman trace class method seems a likely candidate controlling $\sigma_{\text {tot }}$, expecially given the work of Birman and Krein [8] which proves that the T-matrix is trace class and thus Hilbert-Schmidt under suitable hypotheses. In fact, one can control the large coupling constant growth with this method but only under a somewhat stronger hypothesis than (1.1), viz. $\alpha>v$. We do this in Appendix 3. We also note that for central potentials, one can obtain information by a partial wave/phase shift analysis; see Appendix 2. Neither of these methods seems particularly well-suited to multiparticle situations.

Our approach in this paper is motivated by work of Amrein and Pearson [4] (extended to multiparticle situations by Amrein et al. [5]) who use a timedependent approach. One of our initial motivations was to try to make their results more transparent and to analyze various questions left open by them.

We feel we have succeeded in our goal in making the bounds transparent. Our success is based on exploiting recent trends in rigorous scattering theory (see e.g. $[18,45,46,19])$ which exploit $t w o$ related but distinct aspects: time-dependence and geometry. In some sense, we have added geometry to the time-dependent approach of $[4,5]$.

Our basic formula for studying $\sigma_{\text {tot }}$ is :

$$
\|(S-1) g\|^{2}=\int \sigma_{\text {tot }}(k)|\tilde{g}(k)|^{2} d k .
$$


In (1.2), we have a fixed incident direction $\hat{e}$ in mind and $g$ is a function $G$ of $\mathbf{x} \cdot \hat{e}$ alone. $\sigma_{\text {tot }}(k)$ is the total cross-section with initial wave number $k \hat{e}, \tilde{g}$ is the onedimensional Fourier transform, i.e.,

$$
\begin{aligned}
& g(\mathbf{x})=G(\mathbf{x} \cdot \hat{e}) \\
& \tilde{g}(k)=(2 \pi)^{-1 / 2} \int_{-\infty}^{\infty} e^{-i k y} G(y) d y
\end{aligned}
$$

and we require that $\operatorname{supp} \tilde{g} \subset(0, \infty)$. In (1.2) $S$ is the $S$-operator and 1 is the identity operator. Of course, since $g \notin L^{2}$, there is a question of the meaning of " $(S-1) g$ ". We interpret $\|(S-1) g\|$ as

$$
\lim _{R \rightarrow \infty}\left\|(S-1) g h_{R}\right\| \equiv\|(S-1) g\| .
$$

The limit exists for convenient cutoff functions $h_{R}$, e.g.,

$$
h_{R}(\mathbf{x})=\exp \left(-\left[|\mathbf{x} /|^{2}-(\mathbf{x} \cdot \hat{e})^{2}\right] / R^{2}\right) \text {. }
$$

Our fundamental point of view is that (1.2) is a definition of $\sigma_{\text {tot }}$. One can present various "scattering into cones" $[17,28]$ arguments to justify $(1.2)$ from a geometric point of view. An additional point is that one can check (1.2) in twobody situations when one uses the more usual definitions of time-independent scattering theory

$$
\begin{aligned}
& \sigma_{\mathrm{tot}}=\int d \Omega \frac{d \sigma}{d \Omega}, \\
& \frac{d \sigma}{d \Omega}=|f|^{2},
\end{aligned}
$$

with $f$ given in terms of asymptotics of continuum eigenfunctions. This is described in Appendix 1.

Given (1.2), it is easy to estimate $\sigma_{\text {tot }}$; one essentially uses the interaction picture formula for $S$ :

$$
S-1=i\left(\Omega^{-}\right)^{*}\left[\int_{-\infty}^{\infty} d t e^{i t H} V e^{-i t H_{0}}\right]
$$

to obtain

$$
\|(S-1) g\|^{2} \leqq\left(\int_{-\infty}^{\infty}\left\|V e^{-t H_{0}} g\right\| d t\right)^{2} .
$$

In Sect. 2, we use this formula and (1.2) to obtain bounds on $\sigma_{\text {tot }}$ averaged over small intervals in initial momentum. We obtain, up to logarithms, the right borderline for the changeover from finite to infinite $\sigma_{\text {tot }}$ and also the right (i.e., $E^{-1}$ ) large energy behavior. (1.6) has the interpretation of bounding $\sigma_{\text {tot }}$ by adding up all particles scattered out of the beam even those only virtually scattered out. Interference between particles scattered out and the beam is included since we have taken $\left(\int\|\ldots\| d t\right)^{2}$, not $\int\|\ldots\|^{2} d t$. 
In Sect. 3, we exploit the Kupsch and Sandhas [33] idea that

$$
\Omega^{ \pm}=s-\lim _{t \rightarrow \infty} e^{-i t H}(1-j) e^{-i t H_{0}}
$$

for any function of compact support, $j$, so, as in (1.6):

$$
\|(S-1) g\|^{2} \leqq\left(\int_{-\infty}^{\infty}\left\|\left[H(1-j)-(1-j) H_{0}\right] e^{-i t H_{0}} g\right\| d t\right)^{2}
$$

In particular, if $j=1$ on the support of $V$,

$$
\|(S-1) g\|^{2} \leqq\left(\int_{-\infty}^{\infty}\left\|\left[H_{0}, j\right] e^{-i t H_{0}} g\right\| d t\right)^{2} .
$$

$V$ has dropped out of the estimate in (1.8) which allows us to obtain bounds only depending on the size of $\operatorname{supp} V$. We obtain improvements on the results of Amrein and Pearson [4] who used related ideas. (1.8) has the physical interpretation of counting up the particles in the incident beam which enter the region of interaction and suppose all are scattered in order to bound the actual number scattered.

In Sect. 4, we combine the ideas of Sects. 2 and 3 to bound the large $g$ behavior of the cross-section for scattering of $-\frac{1}{2} \Delta+g V$. As we explain in Sect. 5 , the large $g$ behavior is connected with the classical limit where we feel there are interesting open questions. Finally, in Sect. 6, we discuss multiparticle scattering with two initial clusters.

This paper has a number of appendices. The first discusses scattering formulas in general dimension $v$ which are not as one might naively guess since factors of $E^{(v-3) / 4}$ and $E^{(v-3) / 2}$ enter naturally! In particular, we find that in four dimensions the small $E$ behavior of $\sigma_{\text {tot }}$ when there is an $s$-wave resonance is $E^{-3 / 2}(\ln E)^{-2}$. The remaining appendices are not really appendices in the usual sense of containing primarily technical or derivative material. Rather, they apply methods other than the one basic to the paper itself [depending on (1.2)] so we dub them appendices for propagandistic reasons! In Appendix 2, we use Calogero's variable phase method to get lower bounds on the large $g$ behavior of $\sigma_{\text {tot }}$ for scattering from $-\Delta+g V$ which show that the power we find in our upper bounds in Sect. 4 cannot be improved. In Appendix 3, we apply the Birman-Krein theory and, in Appendix 4, Kato's monotonicity theorem.

We end this Introduction with a list of certain open questions which we feel are interesting given what we have done in this paper.

(1) Hard Core Upper Bounds. Let $\mathscr{V}_{R}$ denote the family of positive potentials supported in the ball of radius $R$. For fixed $k$, we almost prove the bound

$$
\sigma_{\text {tot }}(k, V) \leqq c R^{v-1}
$$

for all $v \geqq 3$ and $c$ independent of $R$ and $V \in \mathscr{V}_{R}$ in the sense that in Sect. 4, we prove (1.9) for $\int_{\alpha} \sigma_{\text {tot }}(k, V) d k$ for every $\alpha<\beta$ and $R \geqq R_{0}>0$ (but with $c$ dependent on $\alpha, \beta$, and $R_{0}$ ) and, in Appendix 4, we prove (1.9) for $R$ smaller than some fixed $R_{0}$. Thus, we can define a function

$$
U(k R)=\sup \left\{\left(\tau_{v-1} R^{v-1}\right)^{-1} \sigma_{\text {tot }}(k, V) \mid V \in \mathscr{V}_{R}\right\},
$$


where $\tau_{v-1}$ is the volume of the unit $v-1$ dimensional ball. That $U$ is only a function of $k R$ follows from the scaling relation Theorem A.1.1 in Appendix 1. (1.9), of course, says that

$$
U(k R) \leqq d_{v} .
$$

One question is to verify (1.11) since we have not quite done that because of the necessity of averaging. A much more interesting conjecture we make is the following: Let $H(k R)$ denote $\left(\tau_{v-1} R^{v-1}\right)^{-1} \sigma_{\mathrm{tot}}^{R}(k)$ where $\sigma_{\mathrm{tot}}^{R}(k)$ is the total crosssection from a hard sphere (Dirichlet boundary condition) of radius $R$. One might think that

$$
U(k R) \leqq H(k R)
$$

that is that the largest cross-section occurs for the "largest" $V$ in $\mathscr{V}_{R}$ but as we explain in Sect. 5, the best possible bound for large $k R$ is only

$$
U(k R) \leqq 2 H(k R) .
$$

We remark that in Appendix 4, we prove $\left(1.12^{\prime}\right)$ for $k R$ small and $v \geqq 2$ : this is, however, somewhat special since in that limit $\left(1.12^{\prime}\right)$ holds partial wave by partial wave when $V$ is spherically symmetric and this is certainly false for $k R$ large. We also note that for fixed smooth $V \in \mathscr{V}_{R}, \sigma_{\text {tot }}(k, V) \sim 1 / k^{2}$ for large $k$ while (see Appendix 1)

$$
\lim _{k R \rightarrow \infty} H(k R)=2
$$

and that if (1.11) holds, then (1.12) will hold with $H(k R)$ replaced by $c H(k R)$ for some $c$. Finally, we note that $\left(1.12^{\prime}\right)$ is false for $v=1$ where (with $\tau_{0}=2$ )

$$
H(k R) \equiv 1
$$

but where $U(k R)=2$ once $k R$ is sufficiently large (for $\left[\tau_{0}^{-1} \sigma_{\text {tot }}(k, V)\right]=1-\operatorname{Re} t(k)$ with $t$ the transmission coefficient, $t=0$ for hard core potentials; once $k R$ is sufficiently large, $\operatorname{Re} t=-1$ is allowed). However (1.12) is true in one-dimension; it is an open conjecture in higher dimensions.

(2) Classical Limits and Classical Upper Bounds. Let $\sigma_{\hbar}(p, V)$ denote the total cross-section for incident $k=\hbar^{-1} p$ and for scattering for the pair $\left(-\frac{1}{2} \hbar^{2} \Delta+V\right.$, $-\frac{1}{2} \hbar^{2} \Delta$ ). As we explain in Sect. 5, we believe that

$$
\lim _{h \downarrow 0} \sigma_{h}(p, V)=2 \sigma_{\text {class }}(p, V)
$$

for $V$ sufficiently nice [and finite range; if $V$ is infinite range then (1.14) is presumably true since both sides should be infinite but then the interesting factor of 2 is not meaningful]. (1.14) is an open conjecture. (1.14) can be viewed as an analog of the Weyl type result $[7,36,48,41]$ that the number of bound states approaches classical phase space as $\hbar \downarrow 0$. Motivated by this, one can ask if perhaps the bound

$$
\sigma_{\hbar}(p, V) \leqq \tilde{d}_{v} \sigma_{\text {class }}(p, V)
$$


for all $V$ with $\tilde{d}_{v}$ a $v$-dependent constant. An analogous result holds for the bound state problem $[14,35,42,47]$. (1.15) cannot hold without some restriction. First if $v=2$, it is likely that $\sigma_{h}(p, V) \sim(\ln p)^{2}$ for $p$ small so one should probably only consider (1.15) for $v \geqq 3$. This is a restriction which is also necessary for the bound state result. Moreover, due to problems with $s$-wave resonances (1.15) cannot hold in dimension 3, 4, 5 or 6 if $V$ is allowed to be negative (see the table in Appendix 1). Thus, we only consider (1.15) for $V \geqq 0$ (although it might hold if $v \geqq 7$ for all $V$ ). Finally, (1.15) is false for asymmetric situations. For let $V$ live in a cylinder of radius $R$ and height $L$ with axis along the incident direction. Then $\sigma_{\text {class }}(p, V)$ $=\tau_{v-1} R^{v-1}$ independently of $L$ whereas, as we explain in Sect. 2, $\sigma_{\text {Quantum }}$ surely diverges as $L \rightarrow \infty$. [This does not violate the possibility (1.14), since (1.14) is only to hold for fixed $V$; no uniformity in $V$ is claimed.] Thus, one must look either at $\sigma$ 's averaged over initial angle or at symmetric $V$ 's. Moreover, one can arrange asymmetric $V$ 's where $\sigma_{\text {class }}$ is anomalously small; normally, one expects $\sigma_{\text {class }}$ to be determined by the cross-section of $\operatorname{supp}(V)$, the support of $V$, but, if $V\left(x_{1}, x_{2}, \ldots, x_{v}\right)=f\left(x_{1}\right) W\left(x_{2}, \ldots, x_{v}\right)$ with $W$ constant in a large region and if the initial momentum is in the $(1,0, \ldots, 0)$ direction, then the region where $W$ is constant will not contribute to classical scattering. In fact, we expect in this case that $\lim _{n \rightarrow 0} \sup \sigma_{\hbar}-\lim _{h \rightarrow 0} \inf \sigma_{\hbar}$ is of the order of four times the $y$-1-volume of the region where $W$ is constant. To be conservative, we conjecture that (1.15) holds in $v \geqq 3$ for $V \geqq 0$, spherically symmetric, with geometrically determined cross-section. Notice that for such $V$ 's (1.11) is essentially identical to the conjecture and that $d_{v}=\tilde{d}_{v}$. In Sect. 5, we sketch a possible approach to proving this conjecture.

(3) Sharp E Bounds. Our bounds in Sects. 2-4 only hold for cross-sections averaged over small energy intervals and we have been unable to get bounds on the cross-section at fixed energy. Our attempts to do this have convinced us that in taking the norm inside the integral, in going from (1.5) to (1.6), thereby cancelling $e^{i t H}$, one has lost all hope of controlling sharp energy. It would be good to control these cancellations.

(4) Small E Bounds. In Sect. 2, we prove that for each fixed $V, a, b$

$$
\int_{\mu a}^{\mu b} \sigma_{\text {tot }}(k, V) d k \leqq c(V, a, b) \mu^{-2}
$$

for $\mu$ small. This is more or less saying that we have a bound at low energy diverging as $k^{-3}$. In fact, this cannot be improved uniformly in all dimensions, since an $s$-wave resonance in 4-dimensions leads to a $\sigma_{\text {tot }}$ diverging as $k^{-3}(\ln k)^{-2}$ (see Appendix 1). However, the correct worst case small $k$ behavior is $k^{-1}$ in $v=3$ and $k^{(v-7)}$ for $v \geqq 5$. Can this be obtained with the basic method of this paper? For positive $V$ 's, the correct small $k$ behavior is for $v \geqq 3 k^{(v-3)}$ (see Appendix 1). Can this be obtained with geometric methods? (For $V \geqq 0$ and compact support, we obtain such a bound in Appendix 4 with other methods.)

(5) Small $R$ Bounds. Consider a potential $V$ supported in a sphere of radius $R$ and let $\sigma$ denote the cross-section averaged over a small initial energy range. Then for $c$ depending on $v$, and this energy range we obtain, in Sect. 2, that:

$$
\sigma \leqq c\left(R^{v-1}+R^{v-4}\right)
$$


and for $V \geqq 0$

$$
\sigma \leqq c\left(R^{v-1}+R^{v-2}\right)
$$

When, we announced these results in [20], we conjectured that the $R^{v-2}$ could be dropped in (1.17) and that the $R^{v-4}$ in (1.16) should be $R^{v-3}$. The first conjecture is correct, i.e., using results in Appendix 4, for $V \geqq 0$

$$
\sigma \leqq c R^{v-1}
$$

(see the discussion of problem 1 above). However, in $v=4, \sigma$ can behave as $(\ln R)^{-2}$ for a suitable class of potentials (see Appendix 1). Thus our conjecture in [20] concerning (1.16) is wrong. However, the considerations in Appendix 1 suggest that for arbitrary $V, R \leqq 1$, and $v \geqq 5$;

$$
\sigma \leqq c R^{(2 v-8)}
$$

Can this be proven? $\left(1.16^{\prime}\right)$ is closely related to the $k^{(v-7)}$ behavior conjectured in the discussion of Problem 4.

(6) Atom-Ion Scattering. An induced polarization picture suggests that Coulomb cross-sections with one neutral and one charged cluster will be finite if the neutral system has no static dipole moment. We are unable to prove this. Can one obtain explicit bounds in such a case?

It is a pleasure to thank J. M. Combes, P. Deift, E. Lieb, and W. Thirring, for valuable comments. V. E. would like to thank the Institute for Advanced Study for its hospitality and support under the Albert Einstein visiting professorship endowed by the Federal Republic of Germany and for a travel grant provided by Deutsche Forschungsgemeinschaft.

\section{Basic Estimates in the Two-Body Case}

We begin with an analysis of one-dimensional wave packets.

Lemma 2.1. Let $h_{0}=-\frac{1}{2} d^{2} / d z^{2}$ on $L^{2}(-\infty, \infty)$ and fix a function $G$ in $\mathscr{S}$, the Schwartz space with

$$
\operatorname{supp} \hat{G} \subset(-\delta, \delta)
$$

Let $G_{v}$ be defined by

$$
\hat{G}_{v}(p)=\hat{G}(p-v)
$$

and let $G_{v}(z, t) \equiv\left(e^{-i t h_{0}} G_{v}\right)(z)$. Then:

$$
\left|G_{v}(z, t)\right| \leqq C_{m}[1+|z-v t|]^{-m}
$$

if

$$
z / t \notin[v-\delta, v+\delta]
$$

or

$$
|t| \leqq 1
$$


Moreover,

$$
\left|G_{v}(z, t)\right| \leqq D|t|^{-1 / 2}
$$

for all z, t. In (2.1), $C_{m}$ is a constant depending on $m$ (and $G$ ) but not on $v$.

Proof. Clearly

$$
G_{v}(z)=e^{i z v} G(z)
$$

so, with $p=\frac{1}{i} \frac{d}{d z}$ :

$$
\begin{aligned}
\left|G_{v}(z, t)\right| & =\left|\left[e^{-i z v} e^{-i t h_{0}} e^{i z v} G\right](z)\right| \\
& =\left|\left[\exp \left(-i t\left[e^{-i z v} h_{0} e^{i z v}\right]\right) G\right](z)\right| \\
& =\left|\left[\exp \left(-i t \frac{1}{2}(p+v)^{2}\right) G\right](z)\right| \\
& =\left|\left[\exp \left(-i t h_{0}-i v t p\right) G\right](z)\right| \\
& =|G(z-v t, t)|
\end{aligned}
$$

so it suffices to prove (2.1), (2.2) for $v=0$. (2.1) under hypothesis $\left(2.1^{\prime}\right)$ is then a standard result of integration by parts (see Hörmander [26] or Reed and Simon [40]). (2.2) follows from the fact that $G \in L^{1}$ and $e^{-i t h_{0}}$ is convolution with a function in $L^{\infty}$ with $L^{\infty}$-norm bounded by $C|t|^{-1 / 2}$. For $|t| \leqq 1$, all Schwartz space seminorms of $G_{v}(z, t)$ are uniformly bounded.

Now fix an input direction $\hat{e}$ which we suppose is $(1,0, \ldots)$ and let

$$
h_{R}(\mathbf{x})=\exp \left(-\left(x_{2}^{2}+\ldots+x_{v}^{2}\right) / R^{2}\right) \text {. }
$$

Given $G$, as in Lemma 2.1, let $g_{v}(\mathbf{x})=G_{v}\left(x_{1}\right)$.

Theorem 2.2. Let $H_{0}=-\frac{1}{2} \Delta$. Under the above hypotheses, suppose that $V$ obeys (i) $D(H)=D\left(H_{0}\right)$; (ii) $(1+|\mathbf{x}|)^{1+\varepsilon} V$ is uniformly locally $L^{1}$ for some $\varepsilon>0$. Then:

$$
\sup _{R>1}\left\|(S-1) g_{v} h_{R}\right\| \leqq \int_{-\infty}^{\infty} d t\left(\int_{-\infty}^{\infty}|W(z)|^{2}\left|G_{v}(z, t)\right|^{2} d z\right)^{1 / 2}
$$

where

$$
W(z)=\left[\int\left|V\left(z, x_{2}, \ldots, x_{v}\right)\right|^{2} d x_{2} \ldots d x_{v}\right]^{1 / 2} .
$$

Furthermore $\lim _{R \rightarrow \infty}\left\|(S-1) g_{v} h_{R}\right\|$ exists if the bound (2.3) is finite.

Proof. $g_{v} h_{R}$ lies in $L^{2}$, so, since $\left(\Omega^{-}\right)^{*}$ is a contraction:

$$
\begin{aligned}
\left\|(S-1) g_{v} h_{R}\right\| & =\left\|\left(\Omega^{-}\right)^{*}\left(\Omega^{+}-\Omega^{-}\right) g_{v} h_{R}\right\| \\
& \leqq\left\|\left(\Omega^{+}-\Omega^{-}\right) g_{v} h_{R}\right\| \\
& \leqq \int_{-\infty}^{\infty}\left\|V e^{-i t H_{0}} g_{v} h_{R}\right\| d t
\end{aligned}
$$

(Actually equality holds in (2.5) since asymptotic completeness is known in this case [40].) (2.6) holds because hypotheses (i), (ii) imply that $\left(\Omega^{+}-1\right)$ and $\left(\Omega^{-}-1\right)$ 
exist and when applied to nice vectors like $g_{v} h_{R}$, they can be obtained as integrals of a derivative. Now, write

$$
e^{-i t H_{0}} g_{v} h_{R}=\left(e^{-i t h_{0}} g_{v}\right)\left(e^{-i t \tilde{H}_{0}} h_{R}\right)
$$

and use the elementary Gaussian wave packet calculation:

$$
\sup _{\mathbf{x}, R, t}\left|\left[e^{-i t \tilde{H}_{0}} h_{R}\right](\mathbf{x})\right|=1
$$

to obtain $(2.3)$.

The contribution to the right-hand side of (2.3) is arbitrarily small for large values of $t$ or of $\left(x_{2}, \ldots, x_{v}\right)$, and $\lim _{R \rightarrow \infty}\left[e^{-i t \tilde{H}_{0}} h_{R}\right](\mathbf{x})=1$ uniformly on any bounded region of $t$ - and $\left(x_{2}, \ldots, x_{v}\right)$-values. This implies the existence of the limit.

For the next result we use the norm

$$
\|f\|=\sum_{m=0}^{\infty} \sup _{|n| \geqq m}\left(\int_{n}^{n+1}|f(x)|^{2} d x\right)^{1 / 2} .
$$

Proposition 2.3. Fix $G$. Then for $v \geqq 2 \delta$ the right-hand side of (2.3) is bounded by

$$
c v^{-1}\|W\| \text {. }
$$

Proof. Using $(a+b)^{1 / 2} \leqq a^{1 / 2}+b^{1 / 2}$, we need only separately control the region where (2.1) holds and its complement. Let

$$
u(y)=\left[\int_{-\infty}^{\infty} W(z)^{2}\left(1+|z-y|^{2}\right)^{-1} d z\right]^{1 / 2} .
$$

The contribution from the region of $z$-values where (2.1) holds is bounded by:

$$
C \int_{-\infty}^{\infty} d t u(t v)=C v^{-1} \int_{-\infty}^{\infty} u(y) d y
$$

so a (2.8) type bound follows form

$$
\int_{-\infty}^{\infty} u(y) d y \leqq C\|W\|
$$

But clearly :

$$
|u(y)| \leqq \sum_{n=-\infty}^{\infty} 2\left(1+|y-n|^{2}\right)^{-1}\left(\int_{n}^{n+1}|W(z)|^{2} d z\right)^{1 / 2}
$$

from which (2.9) follows.

By (2.2) and the conditions for (2.1) to hold, the contribution from the region where (2.1) fails is bounded by

$$
D \int_{|t| \geqq 1} d t\left(t^{-1} \int_{(v-\delta) t}^{(v+\delta) t} W(z)^{2} d z\right)^{1 / 2} .
$$


Since for $|t| \geqq 1$

$$
t^{-1} \int_{(v-\delta) t}^{(v+\delta) t}|W(z)|^{2} d z \leqq 2(2 \delta+1) \sup _{|n| \geqq[(v-\delta)|t|]}\left(\int_{n}^{n+1}|W(x)|^{2} d x\right)
$$

we obtain the required bound.

Remarks. In $v$-dimensions if $|V(r)| \leqq C r^{-\alpha}$ at infinity, then the above argument shows that (2.3) and so $\sigma_{\text {tot }}$ is finite so long as $\alpha>\frac{1}{2}(v+1)$ which is exactly the right borderline for $\sigma_{\text {tot }}<\infty$ (see Appendix 2). However, Proposition 2.3 does not get the correct borderline on a logarithmic level, for if

$$
|V(r)| \leqq C r^{-(v+1) / 2}[\log (2+r)]^{-\gamma}
$$

the above estimates require that $\gamma>1$. It is clear that this restriction is not just an artifact of our method of estimating the right-hand side of (2.3). Once we bound

$$
\left\|\int d t e^{i t H} V e^{-i t H_{0}} g\right\|^{2} \text { by }\left(\int\left\|e^{i t H} V e^{-i t H_{0}} g\right\| d t\right)^{2} \text {, }
$$

it is clear that a necessary condition for finiteness is $W \in L^{1}$ which requires $\gamma>1$. As we see in Appendix 2, the correct borderline is $\gamma>\frac{1}{2}$. Note with regard to this kind of borderline that Combes [11] (using Besov space norms) also needs $\gamma>1$, whereas Martin [37] (using Rollnik norms) obtains finiteness for $\gamma>\frac{1}{2}$ if either the norm is small or spherical symmetry holds.

Throwing logarithms to the wind, we state our main result as follows:

Theorem 2.4. Fix $\varepsilon>0$ and let

$$
\|V\|_{F}=\sup _{x}\left[\left(1+\left|x_{1}\right|\right)^{1+\varepsilon} \prod_{i=2}^{v}\left(1+\left|x_{i}\right|\right)^{1 / 2+\varepsilon}\left[\int_{|\mathbf{x}-\mathbf{y}| \leqq 1} V(\mathbf{y})^{2} d^{v} y\right]^{1 / 2}\right] .
$$

Then $\lim _{R \rightarrow \infty}\left\|(S-1) g h_{R}\right\|$ exists for all functions $g$ of the type considered and using (1.2) to define $\sigma_{\text {tot }}$ we have for any $\gamma>0$ and all $v>3 \gamma$ :

$$
\int_{v-\gamma}^{v+\gamma} \sigma_{\text {tot }}(k ; g V) d k \leqq C v^{-2} g^{2}\|V\|_{F}^{2}
$$

for all coupling constants $g$. Here $C$ depends only on $\gamma$ and $\varepsilon$.

Proof. The bound follows from Proposition 2.3 if we pick $G$ so that $\hat{G}=1$ in $(-\gamma, \gamma)$ with say $\delta=\frac{3}{2} \gamma$.

The bound (2.10) has a number of very nice features:

(1) As remarked already except for logs, it has the correct limits in terms of falloff. Notice the asymmetry between the falloff required in directions parallel to the beam direction and those perpendicular.

(2) It has the correct high energy behavior in all dimensions; see Apendix 1 where we examine high energy by using the validity of the Born approximation there.

(3) It has the correct small $g$ behavior, where the Born approximation is applicable. 
(4) It has the "correct" large $g$ behavior in the sense that for any $\mu<2$, there are potentials with $\|V\|_{F}<\infty$ for some $\varepsilon$ so that the left side of (2.10) grows as $g^{\mu}$ as $\mu \rightarrow \infty$; see Appendix 2 .

The deficiencies of the bound (2.10) are the following:

(a) We do not know how to take $\gamma \rightarrow 0$ using only geometric methods. This is the major defect.

(b) No statement has been made about small energy. As we shall see shortly, one can easily say something about small energy but that something is very bad except in dimension 4.

(c) If $V$ has better falloff than borderline, a large $g$ bound better than $g^{2}$ is possible. This is something we remedy in Sects. 3 and 4.

We close this section by noting that using only (2.10) and the scaling relation Theorem A.1.1, one can obtain information about small energy.

Theorem 2.5. If $\|V\|_{F}<\infty$, then for $\lambda \leqq 1$ :

$$
\int_{a \lambda}^{b \lambda} \sigma_{\text {tot }}(k, V) d k \leqq C \lambda^{-2} .
$$

Proof. By Theorem A.1.1:

$$
\begin{aligned}
\int_{a \lambda}^{b \lambda} \sigma_{\text {tot }}(k, V) d k & =\lambda \int_{a}^{b} \sigma_{\text {tot }}(\lambda k, V) d k \\
& =\lambda^{2-v} \int_{a}^{b} \sigma_{\text {tot }}\left(k, V_{\lambda}\right) d k,
\end{aligned}
$$

where $V_{\lambda}(\mathbf{x})=\lambda^{-2} V\left(\lambda^{-1} \mathbf{x}\right)$. But as $\lambda \rightarrow 0$, the sup in the $\left\|V_{\lambda}\right\|_{F}$ norm comes from the $L^{2}$ norm at $\mathbf{x}=0$, so for $\lambda \leqq 1$ :

$$
\left\|V_{\lambda}\right\|_{F}^{2} \leqq c \lambda^{v} \lambda^{-4}
$$

Thus Theorem 2.4 yields (2.11).

Remark. The bound (2.11) suggest a divergence of $\sigma_{\text {tot }}(k, V)$ as $k^{-3}$. As the table in Appendix 1 shows this is not far off in 4 dimensions but is not good in other dimensions. From our geometric point of view, the special nature of 4 dimensions is not apparent.

\section{Potentials of Compact Support}

In the last section, we used the basic Cook method to bound $\sigma_{\text {tot }}$; in this section, we exploit the Kupsch and Sandhas [33] modification of Cook's method to obtain bounds depending only on the size of the support of $V$ but not on the strength of the potential. We emphasis that our results are only mild improvements on those Amrein-Pearson [4] obtained by incorporating the Kupsch and Sandhas method into their frame. We also mention that while our results have the correct large distance behavior, there is room for improvement at short distances: see the discussion in the Introduction. 
Theorem 3.1. Fix $a, b>0$. Then for all $R$,

$$
\int_{a}^{b} \sigma(k \hat{e} ; V) d k \leqq C_{v}\left(R^{v-1}+R^{v-4}\right)
$$

for all $V$ supported in $\{\mathbf{x}|| \mathbf{x} \mid \leqq R\}$ where $C_{v}$ depends only on $v, a, b$.

Remarks. 1. Combining the methods of this and the previous section highly singular potentials and hard cores can be added to the class of potentials considered in Sect. 2 , because the constant $C_{v}$ is independent of $V$. The necessary manipulations are justified if for a suitable smooth cutoff function $j$ (as used in the proof below) $\left(1-j \Psi \in D(H)\right.$ for all $\Psi \in D\left(H_{0}\right)$ and $V(1-j)$ leads to a finite $\|\cdot\| \cdot \|$-norm (2.7).

2. By scaling, for large $\lambda$ (say, $\lambda \geqq 1), \int_{a}^{b} \sigma(\lambda k \hat{e} ; V) d k$ is also bounded by $C_{v}\left(R^{v-1}+R^{v-4}\right)$ (see Appendix 1). Thus these bounds unlike those of Sect. 2 do not have $v^{-2}$ falloff but are uniformly bounded for high energies. This is good since there is no falloff for hard core potentials. If we tried to carry through the energy dependence in our proof, we would find the loss of the $v^{-2}$ factor comes from the appearance of the gradient of $g$ rather than $g$ in (3.2), below.

Proof. Let $j$ be a $C^{\infty}$ function of compact support which is one on the support of $V$. Then

$$
\Omega^{ \pm}=\underset{i \rightarrow \mp \infty}{S-\lim _{t \rightarrow \infty}} e^{i t H}(1-j) e^{-i t H_{0}}
$$

since $j\left(H_{0}+1\right)^{-1}$ is compact. Moreover,

$$
H(1-j)-(1-j) H_{0}=\left[H_{0},(1-j)\right]=-\left[H_{0}, j\right]=\frac{1}{2}(\Delta j)+(\nabla j) \cdot \nabla .
$$

Thus, as in Sect. 2:

$$
\int_{a}^{b} \sigma(k \mathbf{e}, V) d k \leqq\left(\int d t\left[\frac{1}{2}\left\|(\Delta j) e^{-i t H_{0}} g\right\|+\left\|(\nabla j) \cdot e^{-i t H_{0}} \nabla g\right\|\right]\right)^{2},
$$

where $g$ is a function of $\mathbf{x} \cdot \hat{e}$ with $\tilde{g}(k) \equiv 1$ on $(a, b)$. Let $J$ be a function on $C_{0}^{\infty}$ which is 1 for $|\mathbf{x}| \leqq 1$ and let $j=J(\mathbf{x} / R)$. Let

$$
W_{R}(z)=\left[\int\left|\frac{1}{2}(\Delta j)(\mathbf{x})\right|^{2} d^{v-1} x_{\perp}\right]^{1 / 2}
$$

so

$$
W_{R}(z)=R^{(v-5) / 2} W_{1}(z / R) .
$$

By a simple calculation, $\left\|W_{R}\right\|^{2}$ is dominated by $R^{v-3}$ for $R$ large and by $R^{v-4}$ for small $R$. In the same way, the $(\nabla j)$-term is dominated by $R^{v-1}+R^{v-2}$.

Now suppose that $V \geqq 0$, so $\left\|(H+1)^{-1 / 2}\left(H_{0}+1\right)^{1 / 2}\right\| \leqq 1$. By a simple argument using this bound and the compactness of $(H+1)^{-1 / 2}-\left(H_{0}+1\right)^{-1 / 2}$,

$$
\begin{aligned}
\Omega^{ \pm} & =S-\lim _{t \rightarrow \mp \infty} e^{i t H}(H+1)^{-1 / 2}\left(H_{0}+1\right)^{1 / 2} e^{-i t H_{0}} \\
& =\underset{t \rightarrow \lim _{t \rightarrow \infty}}{ } e^{i t H}(H+1)^{-1 / 2}(1-j)\left(H_{0}+1\right)^{1 / 2} e^{-i t H_{0}} .
\end{aligned}
$$


Now we can estimate

$$
\begin{aligned}
& (H+1)^{-1 / 2}\left[H(1-j)-(1-j) H_{0}\right]\left(H_{0}+1\right)^{1 / 2} \\
& =\frac{1}{2}(H-1)^{-1 / 2}\{(\nabla \cdot)(\nabla j)+(\nabla j) \nabla \cdot\}\left(H_{0}+1\right)^{1 / 2}
\end{aligned}
$$

and

$$
\left\|(H+1)^{-1 / 2} \nabla \cdot\right\| \leqq\left\|(H+1)^{-1 / 2}\left(H_{0}+1\right)^{1 / 2}\right\|\left\|\left(H_{0}+1\right)^{-1 / 2} \nabla \cdot\right\| \leqq \sqrt{2} .
$$

Thus, only the $\nabla j$ term in (3.2) remains and so:

Theorem 3.2. Fix $a, b>0$. Then for all $R$ :

$$
\int_{a}^{b} \sigma(k \hat{e} ; V) d k \leqq d_{v}\left(R^{v-1}+R^{v-2}\right)
$$

for all non-negative V's supported in $\{\mathbf{x}|| \mathbf{x} \mid \leqq R\}$.

Remark. If

$$
(H+M) \geqq \alpha\left(H_{0}+M\right)
$$

for some $\alpha>0$, then (3.3) holds with the final $\sqrt{2}$ replaced by $(2 / \alpha)^{1 / 2}$ so that for the class of such $H$ 's [equivalently $V$ 's with $(1-\alpha) H_{0}+V \geqq M(\alpha-1)$ ] an estimate like (3.4) holds but with $d_{v}$ replaced by $d_{v} \alpha^{-1}$. It is interesting to note that the "point potentials" in $v=3$ dimensions, i.e., sequences $V_{R}$ where $\sigma_{\text {tot }}$ has a nonzero limit as $R \rightarrow 0$ have the property that for any $\varepsilon>0, \lim _{R \rightarrow 0} \inf \sigma\left((1-\varepsilon) H_{0}+V_{R}\right)=-\infty$.

\section{Large Coupling Constant}

In Sects. 2 and 3, we considered two "extreme" cases: in Sect. 2, general potentials with $\sigma_{\text {tot }}<\infty$ finding $\sigma_{\text {tot }}(g V) \leqq c g^{2}$ and in Sect. 3, potentials with compact support finding $\sigma_{\text {tot }}(g V) \leqq c$. Here, we will "interpolate" to find $g^{\gamma}$ bounds for $0<\gamma<2$ when $V$ has falloff intermediate between the two extremes. We suppose that for some $\alpha>\frac{1}{2}(v+1)$

$$
|V(\mathbf{x})| \leqq C(1+|\mathbf{x}|)^{-\alpha} \quad|\mathbf{x}| \geqq R_{0}
$$

for some $R_{0}$ and $C$. We will prove:

Theorem 4.1. Let (4.1) hold. Fix $\hat{e}$. Then for $v \geqq 1$, and $g / v$ sufficiently large, we have that

$$
\int_{v-1 / 2}^{v+1 / 2} \sigma_{\text {tot }}(\hat{e}, g V) d k \leqq D(g / v)^{\gamma}
$$

for suitable D, where:

$$
\gamma=(v-1) /(\alpha-1)
$$

Remark. In Appendix 2, we find lower bounds with the same large $g$ behavior as (4.2) for some spherically symmetric $V$ 's obeying (4.1). This shows that the power $\gamma$ of (4.3) is the correct one. 
Proof. For fixed $R \geqq R_{0}$, let $j_{R}$ be $J(\mathbf{x} / R)$ as in the proof of Sect. 3. Since

$$
H\left(1-j_{R}\right)-\left(1-j_{R}\right) H_{0}=\left[j_{R}, H_{0}\right]+V\left(1-j_{R}\right)
$$

we get two terms in estimating $\sigma_{\text {tot }}$. The commutator term is bounded as in Sect. 3, by $C R^{v-1}$. The $V\left(1-j_{R}\right)$ is bounded by a $\|\cdot\|$ of the $W$ associated to $V\left(1-j_{R}\right)$ as in Sect. 2. The net result is

$$
\int_{v-1 / 2}^{v+1 / 2} \sigma_{\mathrm{tot}}(k \hat{e}, g V) d k \leqq C\left(R^{v-1}+v^{-2} g^{2} R^{v+1-2 \alpha}\right) .
$$

Choosing $R=(g / v)^{1 /(\alpha-1)}$, we obtain (4.2).

For exponentially decaying potentials we have the following result:

Theorem 4.2. Let $V(\mathbf{x})$ obey

$$
|V(\mathbf{x})| \leqq C e^{-\mu|\mathbf{x}|} \quad \text { for } \quad|\mathbf{x}| \geqq R_{0},
$$

then for given $\hat{e}$ and any $v \geqq 1, g / v \geqq 2$

$$
\int_{v-1 / 2}^{v+1 / 2} \sigma_{\text {tot }}(k \tilde{e}, g V) d k \leqq D[\ln (g / v)]^{v-1} .
$$

Proof. If the cutoff is made at $R=\left(\mu^{\prime}\right)^{-1} \ln (g / v), \mu^{\prime}<\mu$, the contribution from the outside region is bounded: $((g / v)\|W\|)^{2} \leqq$ const, whereas the inside region is bounded by the right-hand side of (4.5).

\section{Speculations on the Classical Limit}

Fix a potential, $V$. Let $\sigma_{Q}(\mathbf{p}, \hbar)$ denote the total cross-section for scattering involving the pair $\left(-\frac{1}{2} \hbar^{2} \Delta+V ;-\frac{1}{2} \hbar^{2} \Delta\right)$ at incident wave number $\mathbf{k}=\hbar^{-1} \mathbf{p}$. By scaling the time which doesn't change the $S$-operator at all, this is the same as scattering for the pair $\left(-\frac{1}{2} \Delta+\hbar^{-2} V,-\frac{1}{2} \Delta\right)$ at the same value of $k$. Thus, the classical limit $\hbar \downarrow 0$, is a limit where simultaneously we take the coupling constant and the energy to infinity.

In terms of the quantities of the previous section $g \sim \hbar^{-2}, v \sim \hbar^{-1}, g / v \sim \hbar^{-1}$. This makes it intuitively clear why the classical cross-section is generally infinite unless the potential has compact support. Only in the latter case are our bounds independent of $g / v$. [If one wishes one can also scale spatial variables and, using Theorem A.1.1, find that $\sigma_{Q}(\mathbf{p}, \hbar)$ is $\hbar^{\nu-1}$ times the total cross-section for the pair $\left(-\frac{1}{2} \Delta+V(\hbar x),-\frac{1}{2} \Delta\right)$ at wave number $\mathbf{k}=\mathbf{p}$, i.e., we scale the size of the potential outwards and then scale down the size of $\sigma$.]

Similarly, let $d \sigma_{Q} / d \Omega$ be the differential cross-section and let $\sigma_{\mathrm{cl}}(\mathbf{p}), d \sigma_{\mathrm{cl}} / d \Omega$ be the corresponding cross-sections for classical scattering. In this section, we want to explain why we expect that normally one should have:

$$
\lim _{\hbar \downarrow 0} d \sigma_{Q}(p, \hbar) / d \Omega=d \sigma_{\mathrm{cl}}(p) / d \Omega \quad(\Omega \neq(0,0))
$$

and

$$
\lim _{\hbar \downarrow 0} \sigma_{Q}(p, \hbar)=2 \sigma_{\mathrm{cl}}(p) .
$$


We will also sketch a possible proof relying on our basic formula (1.2) which we repeat for easy reference:

$$
\|(S-1) g\|^{2}=\int \sigma_{\mathrm{tot}}(k)|\tilde{g}(k)|^{2} d k .
$$

We use the word "normally" above, since we know of situations where the classical total cross-section is misbehaved under small changes in $V:$ in the usual definition of classical cross-section a piece of phase space with time delay but zero scattering angle contributes nothing to $\sigma_{\mathrm{cl}}$ but for potentials of the type mentioned in Sect. 1, [in the discussion of Problem (2)], there is a positive region of phase space with such behavior so that $\sigma_{\mathrm{cl}}$ will be discontinuous under small changes in $V$. We expect that the classical limit is anomalous in such situations.

The interesting feature of the above is the factor of 2 on the right side of (5.2). Of course, it is only interesting in the case $\sigma_{\mathrm{cl}}<\infty$, so we suppose henceforth in our discussion that $V$ has compact support. Of course, (5.1), (5.2) appear inconsistent with the basic formula

$$
\sigma_{\text {tot }}=\int(d \sigma / d \Omega) d \Omega
$$

In fact, there is no inconsistency because no uniformity is claimed in (5.1). It is our belief that there will be a contribution to $\sigma_{Q}$ of the total magnitude asymptotically exactly equal to $\sigma_{\mathrm{cl}}$ coming from small angles $\theta \lesssim \hbar /|p| R$ (with $R=$ range of $V$ ). This is the celebrated shadow scattering: see Peierls [39] for a discussion of the physics.

Let us first note one case where one can explicitly see that (5.1), (5.2) both hold. Consider a hard core potential of radius $R$, i.e., $V(x)=\infty$ if $|x|<R$ and $=0$ if $|x| \geqq R$. The $\hbar \downarrow 0$ limit is then precisely the same as the $k \rightarrow \infty$ limit where the total cross-section is asymptotically twice the geometric cross-section (see [23] for $v=3$; or Appendix 1 for general $v$; while these arguments are not rigorous, it should be easy to make them rigorous). As explained in Peierls [39], the extra scattering into the shadow, i.e., the difference of $\sigma_{Q}$ and $\sigma_{\mathrm{cl}}$ is all at small angles and it should be possible to easily verify (5.1) in this case.

(5.3) is actually a perfect tool for understanding (and probably also proving) (5.1), (5.2). Suppose that $\operatorname{supp} V=\{\mathbf{x}|| \mathbf{x} \mid<R\}$ so that $\sigma_{\mathrm{cl}}=\pi R^{2}$ under normal circumstances. We first claim that in the $\hbar \downarrow 0$ limit,

$$
\|(S-1) g\| \cong\|(S-1) g h\|,
$$

where $h=h(x, y)$ is one on $x^{2}+y^{2}=\varrho^{2} \leqq R$ and then drops to zero in a region of size $\Delta R$. $\Delta R$ may go slowly to zero as $\hbar \rightarrow 0$. Before giving the proof let us make some heuristic arguments. If $g$ is centered in the region $|z|<R$ at time $t=0$, then $V e^{-i t h_{0}} \mathrm{~g}$ will only be non-negligible (at least in the $\hbar \rightarrow 0$ limit) for times $|t|<2 R / v_{\min } \equiv t_{0}$, where $v_{\text {min }}$ is a minimal velocity in $\hat{g}\left(v_{\min }\right.$ is $\hbar$ independent $)$. Since $h$ has variations over a region of size $\Delta R, \hat{h}$ will have momentum spread over a region of size $\hbar / \Delta R$; so the additional spreading of the step-region, $\Delta s$, during the time $t_{0}$ will be $\Delta s \sim \hbar t_{0} / \Delta R=2 \hbar R / \Delta R$. Thus $\Delta s+\Delta R=O\left(\hbar^{1 / 2}\right)$ if $\Delta R \sim \hbar^{1 / 2}$. This shows that (5.5) should hold for suitable $h$ 's shrinking to the characteristic function of $\{\mathfrak{\varrho}|| \mathbf{\varrho} \mid<R\}$. Let us settle for proving the 
Lemma 5.1. Let $V$ be bounded and supported in $\{\mathbf{x}|| \mathbf{x} \mid<R\}$. Let $H_{0}(\hbar)=-\frac{1}{2} \hbar^{2} \Delta$ and let $h$ be a fixed $C_{0}^{\infty}$ function of $\mathbf{Q}=(x, y, 0)$ which is 1 in $\{\mathbf{g}|| \mathbf{Q} \mid \leqq R\}$. Let $g_{\hbar}$ be a function of $z$ of the form

$$
g_{\hbar}(z)=(2 \pi \hbar)^{-1 / 2} \int e^{i z p / \hbar} \tilde{g}(p) d p
$$

with $\hat{g}$ fixed with support in $\left[v_{\min }, v_{\max }\right] \subset(0, \infty)$. Then

$$
\lim _{\hbar \downarrow 0} \int_{-\infty}^{\infty}\left\|V \exp \left(-i s H_{0}(\hbar)\right)\left[(1-h) g_{\hbar}\right]\right\| d s=0 .
$$

Remarks. 1. As usual (5.6) implies (5.5).

2. We call the variable $s$ in (5.6), since the "proper" time is $t=s \hbar$ although it is (5.6) that enters in estimating $(S-1)$. In looking at the two "time" intervals in the proof, this physically correct variable should be remembered.

3. The proof, slightly extended, shows that the left-hand side of (5.6) is $O\left(\hbar^{N}\right)$ for all $N$.

Proof. Let

$$
g_{\hbar}(z, s)=(2 \pi \hbar)^{-1 / 2} \int e^{i z p / \hbar} e^{-1 / 2 i s p^{2}} \hat{g}(p) d p .
$$

By the usual integration by parts argument (see $[26,40]$ ), one finds that in the region $|z|<R,|s| \geqq 2 R / \hbar v_{\text {min }}$ :

$$
\left|g_{\hbar}(z, s)\right| \leqq C_{N}(\hbar)^{-1 / 2}[\hbar /(|z|+|\hbar s|+\hbar)]^{N}
$$

which shows that the contribution of the region $|s| \geqq 2 R / \hbar v_{\min }$ to (5.6) goes to zero.

Now let $\|\cdot\|_{2}$ denote the $L^{2}\left(d^{2} \varrho\right)$ norm. Then

$$
\left\|\left[\exp \left(-i s H_{0}(\hbar)\right)-1\right](1-h)\right\|_{2} \leqq \frac{1}{2} s \hbar^{2}\|\Delta h\|_{2}
$$

so, since $[V(1-h)]=0$, the contribution to $(5.6)$ in $|s| \leqq C / \hbar$ is $O(\hbar)$.

Remark. From the proof it is clear that Lemma 5.1 remains valid if the cutoff function $h$ is chosen depending on $\hbar$ and converging to a characteristic function if $\hbar\|\Delta h\|_{2} \rightarrow 0$. If $V$ is not central $h$ should be chosen to converge to the characteristic function of the shape of the obstacle as seen by the incident beam. Moreover $|\hat{g}(p)|^{2}$ can be chosen to approach a $\delta$-distribution as $\hbar \rightarrow 0$.

Now in $\|(S-1) g\|$, first replace $g$ by $g h$ where $h$ is very close to the characteristic function of $\{\varrho|| \varrho \mid<R\}$. Next, let $\theta$ be the classical scattering angle as a function of the impact parameter $\mathbf{\varrho}$ so that $\theta(\mathbf{\varrho})=0$ for $\varrho>R$ and suppose $\theta$ is smooth and vanishes in $\varrho<R$ only on a closed set of measure 0 . For given small $\varepsilon$, let $h^{\prime}$ be a smooth approximate of a characteristic function with support in $\{\mathbf{0}|| \theta(\mathbf{0}) \mid>\varepsilon\}$. Then, we can arrange that $\left\|h-h^{\prime}\right\|_{2}$ is very small by taking $\varepsilon$ small and $h$ very close to the characteristic function of a disc. Then, using

$$
\left\|(S-1) g\left(h-h^{\prime}\right)\right\| \leqq 2\left\|h-h^{\prime}\right\|_{2} \int|\hat{g}(p)|^{2} d p
$$

we see that

$$
\int|\hat{g}(p)|^{2} \sigma_{Q}(p, \hbar) d p
$$


will be very close to $\left\|(S-1) g h^{\prime}\right\|$. Now suppose, we can show that in the $\hbar \downarrow 0$ limit, the quantum trajectory $e^{-i t H} g h^{\prime}$ is essentially quasiclassical (with suitable hypotheses on $V$, the method of either [25] or [51] can probably be used to prove this); then (5.1) will follow. In addition, $S g h^{\prime}$ and $g h^{\prime}$ will be orthogonal since, for large times, $e^{-i t H_{0}} g h^{\prime}$ will move in the forward direction while $e^{-i t H_{0}}\left(S g h^{\prime}\right)$ will move in non-forward directions. Thus

$$
\begin{aligned}
\left\|(S-1) g h^{\prime}\right\|^{2} & \simeq\left\|S g h^{\prime}\right\|^{2}+\left\|g h^{\prime}\right\|^{2} \\
& =2\left\|g h^{\prime}\right\|^{2} \\
& \cong 2\left(\pi R^{2}\right) \int|\hat{g}(p)|^{2} d p
\end{aligned}
$$

since the $L^{2}(d \varrho)$ norm of $h^{\prime}$ is very near $\pi R^{2}$ (or the area of the obstacle as seen from the beam direction in the general case).

To summarize, the 2 in (5.2) comes from the fact that we count both the scattered wave $\left\|S g h^{\prime}\right\|^{2}$ and the shadow $\left\|g h^{\prime}\right\|^{2}$ which has been removed in $\|(S-1) g\|^{2}$. Put more forcefully: the formula (5.3) is a wave picture formula and will not be correct in classical mechanics. (This point was emphasized to us especially by E. Lieb.)

One further point about (5.3)/(5.4). If we try to use (5.3) to measure $\sigma_{\text {tot }}$, then in the small $\hbar$-limit we only need a beam whose extent is that of the target; see (5.5). However, if we wanted to use (5.4), we would have to measure $d \sigma / d \Omega$ at angles $\theta \sim \hbar / p R$. To distinguish scattered waves at such small angles from unscattered waves, we have to be able to specify orthogonal momenta in the input beam to much better than $\Delta p \sim \hbar / R$. But that requires a beam width much wider than $R$, i.e., the failure of

$$
\|(S-1) g\|^{2}=\int\left[\int d \Omega(d \sigma / d \Omega)\right]|\hat{g}|^{2} d p
$$

in classical mechanics, is forshadowed in the quasiclassical limit by differences in the required size of the input beam required by the two distinct notions of $\sigma_{\text {tot }}$.

Finally, in terms of the above, we can explain why we asserted in the Introduction that the correct hard core bound might be (1.12) rather than $\left(1.12^{\prime}\right)$. The 2 in (5.2) came from zero interference between $S g h^{\prime}$ and $g h^{\prime}$. But if $S g h^{\prime}$ and $g h^{\prime}$ are antiparallel, then one has $\left\|(S-1) g h^{\prime}\right\|^{2}=4\left\|g h^{\prime}\right\|^{2}$, i.e., a quantum term twice as big as the quasiclassical term even when shadow scattering is included in the quasiclassical term.

Let a plane wave $e^{ \pm i k z}, k=(2 E)^{1 / 2}$, pass a potential well $V(z)=V_{0}>0$ for

$$
O \leqq z \leqq a=\pi\left[(2 E)^{1 / 2}-\left(2\left(E-V_{0}\right)\right)^{1 / 2}\right]^{-1}
$$

and $V(z)=0$ otherwise. If $V_{0}$ is small enough (thus, $a$ is large) the reflected wave is arbitrarily small and the transmitted wave has a phase shifted by $\pi:-e^{ \pm i k z}$ for $z>a$. The same applies for a wave packet $g$ well concentrated around $k$. Let $V(\mathbf{x})$ $=V(z) \chi_{R}\left(x_{2}, \ldots, x_{v}\right), R>a$, and take for $h_{R}$ a smooth approximation of $\chi_{R}$. With $V_{0}$ small and $R$ large enough one can arrange that

$$
\begin{gathered}
\left\{\|(S-1) g\|-\left\|(S-1) g h_{R}\right\|\right\}\left(\tau_{v-1} R^{v-1}\right)^{-1}, \\
\left\{\left\|g h_{R}\right\|\left(\tau_{v-1} R^{v-1}\right)^{-1}-1\right\}
\end{gathered}
$$


and

$$
\left\|(S+1) g h_{R}\right\|\left(\tau_{v-1} R^{v-1}\right)^{-1}
$$

are all smaller than any error $\varepsilon$. Thus the constant in (1.12) cannot be smaller than 2 for $k R$ large.

\section{Two Cluster Multiparticle Scattering}

It is for multiparticle scattering that our formalism really shows its full potential. Here there is no definitive time independent formalism and we will use an analog of our basic (1.2) to define $\sigma_{\text {tot }}$.

We follow the formalism of Sect. XI.5 of [40]: A channel $\alpha$ is a decomposition of $\{1, \ldots, N\}$ into clusters $C_{1}, \ldots, C_{k}$ and bound states $\eta_{1}, \ldots, \eta_{k}$ for each cluster. $\mathscr{H}_{\alpha}$ is the family of functions of differences of the centers of masses of distinct clusters. We define a map $\mathscr{J}_{\alpha}: \mathscr{H}_{\alpha} \rightarrow \mathscr{H}$, the multiparticle Hilbert space by

$$
\mathscr{J}_{\alpha} \phi=\phi \prod_{i=1}^{k} \eta_{i}
$$

i.e., the wave function $\mathscr{J}_{\alpha} \phi$ has the individual clusters in the appropriate bound states and the respective centers of mass in $\phi . H(\alpha)=H-I_{D(\alpha)}$ where $I_{D(\alpha)}$ is the sum of all interactions between distinct clusters $C_{i}$ and $C_{j}$. Finally

$$
\Omega_{\alpha}^{ \pm}=\underset{t \rightarrow \mp-\infty}{S-\lim _{t \rightarrow \infty}} e^{i t H} e^{-i t H(\alpha)} \mathscr{J}_{\alpha}
$$

and

$$
S_{\beta \alpha}=\left(\Omega_{\beta}^{-}\right) * \Omega_{\alpha}^{+}: \mathscr{H}_{\alpha} \rightarrow \mathscr{H}_{\beta} .
$$

We now define the total cross section, $\sigma_{\beta \alpha}$, from a two cluster channel $\alpha$ to any channel $\beta$ by:

$$
\int \sigma_{\beta \alpha}(k)|\tilde{g}(k)|^{2} d k=\left\|\left(S_{\beta \alpha}-\delta_{\beta \alpha}\right) g\right\|_{\beta}^{2}
$$

and the total cross-section $\sigma_{\alpha, \text { tot }}$ by:

$$
\sigma_{\alpha, \text { tot }}(k)=\sum_{\beta} \sigma_{\beta \alpha}(k)
$$

Notice that by the orthogonality of channels (Theorem XI.36(b) of [40]):

$$
S_{\beta \alpha}-\delta_{\beta \alpha}=\left(\Omega_{\beta}^{-}\right)^{*}\left(\Omega_{\alpha}^{+}-\Omega_{\alpha}^{-}\right)
$$

and also

$$
\sum_{\beta}\left\|\left(\Omega_{\beta}^{-}\right)^{*} u\right\|^{2} \leqq\|u\|^{2}
$$

[Equality will hold in (6.4) for $u \in \mathscr{H}_{\mathrm{ac}}(H)$, if asymptotic completeness holds.] Combining (6.1)-(6.4) we see that

$$
\int \sigma_{\alpha, \mathrm{tot}}(k)|\tilde{g}(k)|^{2} d k \leqq\left\|\left(\Omega_{\alpha}^{+}-\Omega_{\alpha}^{-}\right) g\right\|^{2} .
$$


By the usual Cook argument

$$
\left\|\left(\Omega_{\alpha}^{+}-\Omega_{\alpha}^{-}\right) g\right\| \leqq \int_{-\infty}^{\infty}\left\|I_{D(\alpha)} e^{-i t H(\alpha)} g \prod_{1}^{2} \eta_{i}\right\| d t .
$$

Letting $h_{0}(\alpha)$ denote the kinetic energy of relative motion of the clusters in $\alpha$ and $\|\cdot\|_{\alpha}$ the $\mathscr{H}_{\alpha}$ norm, we have proven that:

Theorem 6.1. In multiparticle systems :

$$
\int \sigma_{\alpha, \text { tot }}(k)|\tilde{g}(k)|^{2} d k \leqq\left(\int_{-\infty}^{\infty}\left\|V_{\text {eff }} e^{-i t h_{0}(\alpha)} g\right\| d t\right)^{2},
$$

where

$$
V_{\text {eff }}\left(\mathbf{R}_{\alpha}\right)=\left[\int\left|I_{D(\alpha)}\left(\mathbf{R}_{\alpha}, \zeta\right)\right|^{2}\left|\eta_{1}\left(\zeta_{1}\right)\right|^{2}\left|\eta_{2}\left(\zeta_{2}\right)\right|^{2} d \zeta_{1} d \zeta_{2}\right]^{1 / 2} .
$$

The effective potential (6.7) is to be distinguished from the function

$$
\tilde{V}_{\text {eff }}\left(\mathbf{R}_{\alpha}\right)=\int I_{D(\alpha)}\left(\mathbf{R}_{\alpha}, \zeta\right)\left|\eta_{1}\left(\zeta_{1}\right)\right|^{2}\left|\eta_{2}\left(\zeta_{2}\right)\right|^{2} d \zeta_{1} d \zeta_{2}
$$

which often enters in other contexts see e.g., $[32,46,44]$. Cancellations are more efficient in $\tilde{V}_{\text {eff }}$, e.g., for $C_{1}=$ proton and electron and $C_{2}$ a single electron with the usual Coulomb forces, $\tilde{V}_{\text {eff }}$ is $O\left(e^{-c R_{\alpha}}\right)$ while $V_{\text {eff }}\left(R_{\alpha}\right)$ is $O\left(R_{\alpha}^{-2}\right)$ at infinity.

By the method of Sect. 2, we clearly have

Corollary 6.2. If each $V_{i j}(\mathbf{r})$ for $i, j$ in distinct clusters obeys:

$$
\left|V_{i j}(\mathbf{r})\right| \leqq C(1+|\mathbf{r}|)^{-\alpha}
$$

with $\alpha>\frac{1}{2}(v-1)$, then $\sigma_{\text {tot }}<\infty$;

and also

Corollary 6.3. $(v=3)$. If each $V_{i j}$ is Coulomb, i.e., $V_{i j}(r)=e_{i} e_{j}|r|^{-1}$ and if both clusters are neutral (atom-atom scattering), then $\sigma_{\text {tot }}<\infty$.

The point is that if both clusters are neutral, then $V_{\text {eff }}\left(R_{\alpha}\right) \sim R_{\alpha}^{-3}$ at infinity. This is not changed if there are short range potentials in addition to Coulomb potentials. However, if cluster 2 is charged but cluster 1 is neutral, then $V_{\text {eff }}\left(R_{\alpha}\right)$ $\sim c R_{\alpha}^{-2}$ at infinity with

$$
c^{2}=\int\left|\eta_{1}\left(\zeta_{1}\right)\right|^{2}\left|\eta_{2}\left(\zeta_{2}\right)\right|^{2}\left|\sum_{\substack{i \in C_{1} \\ j \in C_{2}}} e_{i} e_{j} \hat{R} \cdot\left(\zeta_{(i)}-\zeta_{(j)}\right)\right|^{2} d \zeta_{1} d \zeta_{2}
$$

with $\zeta_{(i)}$ the vector from $\mathbf{r}_{i}$ to the center of mass of cluster 1 . For example, when $C_{2}$ has a single charged particle and $C_{1}$ has two of opposite charge, then

$$
c^{2}=d \int\left|\eta_{1}\left(\mathbf{r}_{12}\right)\right|^{2}\left|\hat{R} \cdot \mathbf{r}_{12}\right|^{2} d^{3} r_{12},
$$

where $d$ is a function of the mass ratio of particles 1,2 and the charges. In this case $c \neq 0$ for any direction $\hat{R}$.

Thus our method is capable of accommodating atom-atom scattering but not atom-ion scattering (by estimating $\tilde{V}_{\text {eff }}$ rather than $V_{\text {eff }}$, we made an erroneous claim about this in [20]). We expect $\sigma_{\text {tot }}<\infty$ for atom-ion scattering if the atom has no static dipole moment; as usual the limitation comes from replacing $\left\|\int \ldots\right\|^{2}$ by $\left(\int\|\|\right)^{2}$. 


\section{Appendix 1. Two-Body Scattering in v-Dimensions}

In this appendix, we want to consider mostly formal aspects of the $v$ dependence of scattering for $H=-\Delta+V$ on $L^{2}\left(R^{v}\right)$. Among other things, we want to establish (1.2) in cases where the kernel of $S-1$ is sufficiently nice. We note that while we deal with $-\frac{1}{2} \Delta+V$ in the body of the paper, establishing (1.2) for $-\Delta+V$, establishes it also for $-\alpha \Delta+V$ by scaling time (and replacing $V$ by $\alpha^{-1} V$ ). For rigorous results on Lippman-Schwinger equations, etc. in $v$-dimensions see Thoe [49] and Alsholm and Schmidt [3].

There is an eigenfunction transform based on functions $\phi(\mathbf{x}, \mathbf{k})$ obeying

$$
\phi(\mathbf{x}, \mathbf{k})=e^{i \mathbf{k} \cdot \mathbf{x}}-\int G_{0}(\mathbf{x}-\mathbf{y}, \mathbf{k}) V(\mathbf{y}) \phi(\mathbf{y}, \mathbf{k}) d y,
$$

where $G_{0}$ is the kernel of $\left(-\Delta-k^{2}-i o\right)^{-1}$. We will need the following formula for large $x[3]$ :

$$
\begin{aligned}
G_{0}(x, k) \sim & k^{(v-3) / 2} \pi^{-(v-1) / 2} 2^{-(v+1) / 2} e^{-i(\pi / 4)(v-3)} \\
& \cdot|x|^{-(v-1) / 2} e^{i k|x|} .
\end{aligned}
$$

From this we read off the asymptotics of the Lippman-Schwinger function

$$
\phi(\mathbf{x}, \mathbf{k}) \sim e^{i \mathbf{k} \cdot \mathbf{x}}+f\left(\mathbf{k}^{\prime}, \mathbf{k}\right) e^{i k r_{r}-(v-1) / 2},
$$

where $r=|\mathbf{x}| \rightarrow \infty$ and $\mathbf{k}^{\prime}=|\mathbf{k}| \mathbf{x} r^{-1}$ fixed. In (A.1.3), $f$ is given by:

$$
f\left(\mathbf{k}^{\prime}, \mathbf{k}\right)=-k^{(v-3) / 2} \pi^{-(v-1) / 2} 2^{-(v+1) / 2} e^{-i(\pi / 4)(v-3)}(2 \pi)^{v} T\left(\mathbf{k}^{\prime}, \mathbf{k}\right)
$$

with $T$ the usual $T$ matrix defined by

$$
T\left(\mathbf{k}, \mathbf{k}^{\prime}\right)=(2 \pi)^{-v} \int e^{-i \mathbf{k} \cdot \mathbf{y}} V(\mathbf{y}) \phi\left(\mathbf{y}, \mathbf{k}^{\prime}\right) d y .
$$

The differential cross section is defined as usual from (A.1.3) by:

$$
\frac{d \sigma}{d \Omega}\left(k \rightarrow k^{\prime}\right)=\left|f\left(\mathbf{k}^{\prime}, \mathbf{k}\right)\right|^{2}
$$

so that by (A.1.4)

$$
\frac{d \sigma}{d \Omega}=k^{(v-3)} \pi^{(v+1)} 2^{(v-1)}\left|T\left(\mathbf{k}^{\prime}, \mathbf{k}\right)\right|^{2}
$$

As in the three-dimensional case $[40,27]$, one has the relation [49]:

$$
(S-1)\left(\mathbf{k}, \mathbf{k}^{\prime}\right)=-2 \pi i T\left(\mathbf{k}, \mathbf{k}^{\prime}\right) \delta\left(k^{2}-\left(k^{\prime}\right)^{2}\right)
$$

for the kernel of the $S$-operator.

Now let $g$ be a function of a single variable viewed as a function on $R^{v}$. If $\hat{g}$ is the $v$-dimensional Fourier transform and $\tilde{g}$ is the one-dimensional Fourier transform, then

$$
\hat{g}(\mathbf{k})=\tilde{g}(|\mathbf{k}|) \delta\left(\mathbf{k}_{\perp}\right)(2 \pi)^{(v-1) / 2} .
$$

Thus, using (A.1.7):

$$
\left(\widehat{S-1) g}(\mathbf{k})=(-2 \pi i)(2|\mathbf{k}|)^{-1} T(\mathbf{k},|\mathbf{k}| \hat{e}) \tilde{g}(|\mathbf{k}|)(2 \pi)^{(v-1) / 2}\right.
$$


with $\hat{e}$ the direction of $g$. For (A.1.8) to be true we require that $\tilde{g}$ have support in $(0, \infty)$. Thus, by (A.1.6):

$$
\begin{aligned}
\|(S-1) g\|^{2} & =\int\left[\int d \Omega_{\hat{e}^{\prime}}\left|T\left(k \hat{e}^{\prime}, k \hat{e}\right)\right|^{2}\right](2 \pi)^{v+1}(2 k)^{-2}|\tilde{g}(k)|^{2} k^{v-1} d k \\
& =\int\left[\int \frac{d \sigma}{d \Omega} d \Omega\right]|\tilde{g}(k)|^{2} d k \\
& =\int \sigma_{\text {tot }}(k)|\tilde{g}(k)|^{2} d k
\end{aligned}
$$

as claimed in (1.2).

One can read the high energy behavior of $\sigma_{\text {tot }}$ off of (A.1.4)/(A.1.6). In great generality, the Born approximation is valid at large $|k|[52,21]$, i.e.,

$$
T\left(k, k^{\prime}\right) \sim \hat{V}\left(k-k^{\prime}\right)
$$

for $\left|k^{\prime}\right|$ large. Since $\left|k-k^{\prime}\right|^{2}=2 k^{2}(1-\cos \theta)$ in polar coordinates with $|k|=\left|k^{\prime}\right|$ (A.1.9) tells us that

$$
\int\left|T\left(k \hat{e}^{\prime}, k \hat{e}\right)\right|^{2} d \Omega_{\hat{e}^{\prime}} \sim c k^{-(v-1)}
$$

since $T$ is only non-negligible in a $(v-1)$ dimensional region of radius $\sim k^{-1}$. Thus, by (A.1.6), we see that

$$
\sigma_{\text {tot }} \sim c k^{-2} ; k \rightarrow \infty \text {, any } v
$$

which is exactly the high energy behavior of our bound in the bulk of the paper.

The low energy behavior is more subtle and has a more interesting vdependence. Let us suppose that $V \leqq 0$ and, since we are not worrying about optimal conditions in this appendix, suppose that $V \in C_{0}^{\infty}$. Then the operator

$$
Q(k)=|V|^{1 / 2}\left(-\Delta-k^{2}-i O\right)^{-1}|V|^{1 / 2}
$$

is compact. Moreover, we have that

$$
T\left(k, k^{\prime}\right)=-(2 \pi)^{-v}\left\langle|V|^{1 / 2} e^{i k \cdot x},(1-Q(k))^{-1}|V|^{1 / 2} e^{i k^{\prime} \cdot x}\right\rangle .
$$

Let us suppose $v \geqq 3$, since for $v=1,2, Q(0)$ is not defined and the analysis is much harder [31]. If $1 \notin \sigma(Q(0))$, then $T$ is bounded as $k \rightarrow 0$ and in that case

$$
\sigma_{\text {tot }}(k) \sim k^{(v-3)}, \quad(1 \notin \sigma(Q(0)) .
$$

The same applies for $V \geqq 0$ because $(1+Q(k))^{-1}$ which then arises in (A.1.11) is always bounded.

Consider next the case where $1 \in \sigma(Q(0))$ and suppose that 1 is a simple eigenvalue (we will remark on what happens if it is not later). The $k$ dependence of such eigenvalues has been extensively analyzed recently by Klaus and Simon [31], who study behavior of eigenvalues at absorption into continua, and by Jensen [29], who studies large time behavior. They find that if $\mu(0)=1$ is a simple eigenvalue $(c \neq 0)$ :

$$
\begin{aligned}
\mu(k) & =1+c k^{2}+\ldots & & (v \geqq 5) \\
& =1+c k^{2} \ln k+\ldots & & (\text { Case A } ; v=4) \\
& =1+c k+\ldots & & (\text { Case } \mathrm{A} ; v=3) .
\end{aligned}
$$


In addition, in $v=3,4$, the behavior (A.1.13) can occur which we call Case B.

If $\eta$ is the normalized vector with $Q(0) \eta=\eta$, then (A.1.11) implies that

$$
T\left(k, k^{\prime}\right)=(2 \pi)^{-v}(1-\mu(k))^{-1}\left\{\left|\left\langle|V|^{1 / 2}, \eta\right\rangle\right|^{2}+O(k)\right\} .
$$

In all the cases we have labelled Case $\mathrm{A},\left\langle|V|^{1 / 2}, \eta\right\rangle \neq 0$ and in the other cases $\left\langle|V|^{1 / 2}, \eta\right\rangle=0$ (either case is possible in $v \geqq 5$ ). It follows that Case A, which we call the case of " $s$-wave dominance" is always the most singular case. Moreover [31], since Case $\mathrm{A}$ is always a non-degenerate eigenvalue, there is no loss in our assumption of simplicity. We summarize the situation in the table below:

Table A.1

\begin{tabular}{ll}
\hline$v$ & $\sigma_{\text {tot }}$ in $s$-wave dominance case for $E$ small \\
\hline 3 & $E^{-1}$ \\
4 & $E^{-3 / 2}(\ln E)^{-2}$ \\
5 & $E^{-1}$ \\
6 & $E^{-1 / 2}$ \\
7 & const \\
$\geqq 8$ & 0 \\
\hline
\end{tabular}

Thus, the small $E$ behavior we obtain with our geometric bounds cannot be improved in general dimension by more than a logarithm. In dimension $v \neq 4$, there is considerable room for improvement.

Next, we want to look at scaling dependence:

Theorem A.1.1. Let $V_{\lambda}(\mathbf{x})=\lambda^{-2} V\left(\lambda^{-1} \mathbf{x}\right)$. Then

$$
\sigma_{\text {tot }}\left(k ; V_{\lambda}\right)=\lambda^{(v-1)} \sigma_{\text {tot }}(\lambda k ; V),
$$

where $\sigma_{\text {tot }}(k, W)$ is the total cross section for $-\Delta+W$ at incident momentum ke $(\hat{e}$ fixed).

Proof. While there are various methods available; we will use our basic formula (1.2). Let

$$
(U(\lambda) f)(\mathbf{x})=\lambda^{-v / 2} f\left(\lambda^{-1} x\right)
$$

be the unitary scale transformation. Then, with $H_{0}=-\Delta$ :

$$
U(\lambda) H U(\lambda)^{-1}=\lambda^{2}\left(H_{0}+V_{\lambda}\right)
$$

so

$$
\begin{aligned}
U(\lambda) \Omega^{ \pm}\left(H, H_{0}\right) U(\lambda)^{-1} & =\Omega^{ \pm}\left(\lambda^{2}\left(H_{0}+V_{\lambda}\right), \lambda^{2} H_{0}\right) \\
& =\Omega^{ \pm}\left(H_{0}+V_{\lambda}, H_{0}\right)
\end{aligned}
$$

by scaling time. Thus

$$
U(\lambda)[S(V)-1] U(\lambda)^{-1}=\left[S\left(V_{\lambda}\right)-1\right] .
$$

Notice next that

$$
\begin{aligned}
\left(U(\lambda)^{-1} g\right)^{\sim}(k) & =(2 \pi)^{-1 / 2} \int \lambda^{+v / 2} g(\lambda z) e^{i k z} d z \\
& =\lambda^{(v-2) / 2} \tilde{g}\left(\lambda^{-1} k\right) .
\end{aligned}
$$


Thus, using (A.1.17) and (1.2):

$$
\begin{aligned}
\int \sigma_{\text {tot }}\left(k ; V_{\lambda}\right)|\tilde{g}(k)|^{2} d k & =\left\|(S(V)-1) U(\lambda)^{-1} g\right\|^{2} \\
& =\int \sigma_{\text {tot }}(k ; V) \lambda^{(v-2)}\left|\tilde{g}\left(\lambda^{-1} k\right)\right|^{2} d k \\
& =\int \lambda^{\nu-1} \sigma_{\text {tot }}(\lambda k ; V)|\tilde{g}(k)|^{2} d k .
\end{aligned}
$$

Since $\tilde{g}$ is arbitrary, the theorem is proven.

Now, with $v=3,4$, fix $V$ a potential with support in $\{x|| x \mid \leqq 1\}$ with an $s$-wave zero energy resonance and consider the sequence of potentials $V_{\lambda}$ with $\lambda \rightarrow 0$ (when $v=3$, this is the "standard" point potential in the limit $[22,2,50,24])$. Fix also $k \neq 0$. The small $\lambda$ limit of $\sigma_{\text {tot }}\left(k, V_{\lambda}\right)$ can be read off the small $k$ limit of $\sigma_{\text {tot }}(k ; V)$ in Table A.1; if $v=4,3$ we have (replacing $\lambda$ by $R$ ):

$$
\begin{array}{cc}
(\ln R)^{-2} & (v=4) \\
(\text { const }) & (v=3) .
\end{array}
$$

The $v=4$ case proves that again for general $v$, our $R^{v-4}$ result can only be marginally improved. For $v \neq 4$, there is considerable room for improvement.

Finally, we want to consider the eigenshift expansion (i.e., the relation between $\sigma_{\text {tot }}$ and the eigenvalues of the on-shell $S$-matrix), and use it to study the behavior of the hard core scattering cross section. By (A.1.7), the on-shell $S$-matrix has an integral kernel given by:

$$
(S(E)-1)\left(\Omega, \Omega^{\prime}\right)=-\pi i(E)^{(v-2) / 2} T\left(E^{1 / 2} \Omega, E^{1 / 2} \Omega^{\prime}\right)
$$

so, by (A.1.6):

$$
\frac{d \sigma}{d \Omega}\left(\Omega \leftarrow \Omega^{\prime}\right)=k^{-(v-1)}(2 \pi)^{v-1}\left|(S(E)-1)\left(\Omega, \Omega^{\prime}\right)\right|^{2} .
$$

Thus, letting

$$
\sigma_{a v}=\left(S_{v-1}\right)^{-1} \int \frac{d \sigma}{d \Omega}\left(\Omega \leftarrow \Omega^{\prime}\right) d \Omega d \Omega^{\prime}
$$

with $S_{v-1}$ the area of the $v-1$ dimensional sphere, we have

$$
\sigma_{a v}=(2 \pi)^{v-1}\left(S_{v-1}\right)^{-1} k^{-(v-1)}\|S(E)-1\|_{2}^{2}
$$

with $\|\cdot\|_{2}$ the Hilbert-Schmidt norm. If $S(E)$ has eigenvalues $e^{2 i \delta_{\alpha}(E)}$ counting multiple eigenvalues, a multiple number of times, we have that

$$
\sigma_{a v}=(2 \pi)^{v-1}\left(S_{v-1}\right)^{-1} k^{-(v-1)} \sum_{\alpha}\left|e^{2 i \delta_{\alpha}(E)}-1\right|^{2} .
$$

If $V$ is spherically symmetric, using the multiplicity of the spherical harmonics, we find that

$$
\sigma(E)=(2 \pi)^{v-1}\left(S_{v-1}\right)^{-1} k^{-(v-1)} \sum_{\ell=0}^{\infty}(2 \ell+v-2) \frac{(\ell+v-3) !}{(v-2) ! \ell !} 4 \sin ^{2} \delta_{\ell} .
$$


Consider now hard core scattering when $v \geqq 2$. In the high energy limit (see [23, Problem 109 for $v=3]$ ) for small $\ell, \sin ^{2} \delta_{\ell}$ alternates between being approximately $\sin ^{2} k R$ and $\cos ^{2} k R$ such that we replace it by its average value $1 / 2$. Moreover, there is effectively a sharp cutoff at $\ell \sim k R$. Thus,

$$
\begin{aligned}
\sum_{\ell=0}^{\infty}(2 \ell+v-2) \frac{(\ell+v-3) !}{(v-2) ! \ell !} 4 \sin ^{2} \delta_{\ell} & \cong 2 \sum_{\ell=0}^{[k R]}(2 \ell+v-2) \frac{(\ell+v-3) !}{(v-2) ! \ell !} \\
& \cong \frac{2}{(v-2) !} \int_{0}^{k R} 2(\ell)^{v-2} d \ell \\
& \cong \frac{4}{(v-1) !}(k R)^{v-1} .
\end{aligned}
$$

If $\tau_{v}$ is the volume of the unit ball in $v$-dimensions, we have that

$$
S_{v-1} \tau_{v-1}=2(2 \pi)^{v-1} /(v-1) !
$$

so that the last calculation and (A.1.19c) lead to

$$
\sigma_{R}(E) \sim 2 \tau_{v-1} R^{v-1} \quad(k R \rightarrow \infty ; \text { hard core }) .
$$

This is the expected geometric plus shadow scattering.

At intermediate energies (A.1.19c) tells us that $\sigma_{R}(E) \sim k^{-(v-1)} f(k R)$ in agreement with the scaling relation Theorem A.1.1. Finally, to obtain the low energy result, we note that when $(v, \ell) \neq(2,0)$,

$$
\tan \left[\delta_{\ell}^{v}(k, R)\right]=j_{\ell^{\prime}}(k R) / n_{\ell^{\prime}}(k R)
$$

with $\ell^{\prime}=\ell+\frac{1}{2}(v-3)$ so that for $k R$ small

$$
\delta_{\ell}^{v}(k, R) \sim c_{\ell, v}(k R)^{2 \ell^{\prime}+1} .
$$

So for $v \neq 2$, the leading $\ell=0$-term is

$$
\delta_{\ell=0}^{v}(k, R) \sim c_{0, v}(k R)^{(v-2)}
$$

and so by $($ A.1.19c) $(v \geqq 3)$ :

$$
\sigma_{R}(E) \sim c_{v} k^{v-3} R^{2 v-4} \quad(k R \rightarrow 0)
$$

a careful calculation shows that

$$
c_{v}=(2 \pi)^{v-1}\left(S_{v-1}\right)^{-1}\left\{\frac{2^{(v-2)}[(v-3 / 2) !]^{2}}{(v-3) !(v-2) !}\right\}^{2}
$$

which is the familiar $4 \pi$ when $v=3]$. Comparing (A.1.20) and (A.1.12), we see that hard and "soft" repulsive potentials have the same small $k$ behavior which is reasonable. The only surprise is that the $R^{2}$ behavior in $v=3$ dimensions might mislead one to think $R^{v-1}$ was the small $R$ behavior as it is for large $R$. Note that only if $v=3$ does $v-1=2 v-4$. 


\section{Appendix 2. The Variable Phase Method and Lower Bounds on $\sigma_{\text {tot }}$}

In this appendix, our main goal is to prove

Theorem A.2.1. Let $V$ be a central potential on $R^{3}$ obeying $(\alpha>2)$ :

$$
(1+r)^{-\alpha} \leqq V(r) \leqq A(1+r)^{-\alpha}
$$

Then, for any fixed energy, the total cross section $\sigma_{\text {tot }}(E, g)$ for $-\Delta+g V$ obeys:

$$
\sigma_{\text {tot }}(E, g) \geqq c g^{2 /(\alpha-1)}
$$

for all sufficiently large $g$.

Remarks. 1. The same method proves an upper bound of the form $D g^{2 /(\alpha-1)}$. Of course, unlike the method in the paper, this proof of the upper bound requires central V's.

2. The same method in $v$-dimensions yields a $g^{(v-1) /(\alpha-1)}$.

3. The constants are uniform in $k$ running over intervals, so averaged cross sections have the same behavior.

We will also prove:

Theorem A.2.2. Let $V$ be a central potential on $R^{3}$ obeying (A.2.1) with $1<\alpha \leqq 2$. Then $\sigma_{\text {tot }}(E)=\infty$ for any $g$.

Remark. 1. In $v$-dimensions, the same argument works with $\alpha \geqq \frac{1}{2}(v+1)$.

2. The same arguments show that if $(1+r)^{-\alpha}$ in (A.2.1) is replaced by $(1+r)^{-2}[\log (2+r)]^{-\gamma}$ (or $(1+r)^{-(v-1) / 2}[\log (2+r)]^{-\gamma}$ in $v$ dimensions), then $\sigma_{\text {tot }}$ is finite if $\gamma>\frac{1}{2}$ and infinite if $\gamma \leqq \frac{1}{2}$.

Both theorems depend on controlling the asymptotics of the partial wave phase shift $\delta_{\ell}(g)$ which we will show behaves like $-g \ell^{-(\alpha-1)}$ for $\ell$ large, uniformly in $g$. In the region where $\delta_{\ell}$ is small, we can replace $\sin ^{2} \delta_{\ell}$ by $\delta_{\ell}^{2}$. If $\alpha \leqq 2$, the series $\sum(2 \ell+1) \delta_{\ell}^{2}$ will diverge and if $\alpha>2$, the series $c \sum_{g \ell-(\alpha-1)<\varepsilon}(2 \ell+1) \delta_{\ell}(g)^{2}$ will give the lower bound (A.2.2). Our control of $\delta_{\ell}$ will depend on the "variable phase" method developed by Calogero [10] and Babikov [6]. According to them

$$
\delta_{\ell}(g)=\lim _{r \rightarrow \infty} d_{\ell}(g, r)
$$

where $d_{\ell}(g, r)$ obeys (for simplicity, we work with $k=1$ )

$$
d_{\ell}^{\prime}(r)=-g V(r) \hat{D}_{\ell}(r)^{2} \sin ^{2}\left(\hat{\delta}_{\ell}(r)+d_{\ell}(r)\right)
$$

The precise boundary conditions are unimportant, except that for $V \geqq 0$, they imply [10, p. 15]

$$
O \leqq-d_{\ell}(r) \leqq \hat{\delta}_{\ell}(r)
$$

In (A.2.4), the functions $\hat{D}_{\ell}, \hat{\delta}_{\ell}$ are connected with Bessel functions. For our purposes, the following formulae [10, pp. 200, 201] are most useful:

$$
\begin{gathered}
\hat{\delta}_{\ell}(x)=\int_{0}^{x} d z \hat{D}_{\ell}^{-2}(z), \\
\hat{D}_{\ell}^{2}(x)=\frac{4 x}{\pi} \int_{0}^{\infty} d t \cosh ((2 \ell+1) t) K_{0}(2 x \sinh t),
\end{gathered}
$$


where $K_{0}$ is the standard Bessel function of imaginary argument. We will also need the formula $[10$, p. 200]:

$$
\hat{D}_{\ell}^{2}(x)=\sum_{n=0}^{\ell} x^{-2 n}\left[\frac{(\ell+n) !}{(\ell-n) !} \frac{(2 n-1) ! !}{2 n ! !}\right]
$$

Lemma A.2.3. (i) $\hat{D}_{\ell}^{2}(x)$ is monotone decreasing in $x$ for $\ell$ fixed and

$$
\hat{D}_{\ell}^{2}(x) \geqq \lim _{x \rightarrow \infty} \hat{D}_{\ell}^{2}(x)=1 \text {. }
$$

(ii) For any fixed $y, \hat{D}_{\ell}^{2}((2 \ell+1) y)$ is increasing in $\ell$ and

$$
g(y)=\lim _{\ell \rightarrow \infty} \hat{D}_{\ell}^{2}(y(2 \ell+1))
$$

is finite if, and only if, $y>\frac{1}{2} . g(y)$ is monotone decreasing, $\lim _{y \rightarrow \infty} g(y)=1$ and for $y$ near $\frac{1}{2}$

$$
\begin{array}{r}
g(y) \leqq C\left(y-\frac{1}{2}\right)^{-1 / 2} \\
\text { (iii) } \lim _{\ell \rightarrow \infty}(2 \ell+1)^{-1} \hat{D}_{\ell}^{2}\left(\frac{1}{2}(2 \ell+1)-\sqrt{2 \ell+1}\right)=\infty .
\end{array}
$$

Proof. (i) Follows immediately from (A.2.8).

(ii) By a change of variables in (A.2.7) $(t=s / 2 x)$ :

$$
\hat{D}_{\ell}^{2}((2 \ell+1) y)=\frac{2}{\pi} \int_{0}^{\infty} d s \cosh (s / 2 y) K_{0}\left(s f\left(\frac{s}{2 y(2 \ell+1)}\right)\right)
$$

where $f(u)=u^{-1} \sinh u$. As $\ell$ increases, $u \equiv s / 2 y(2 \ell+1)$ decreases, so

$$
f(u)=u^{-1} \int_{0}^{u} \cosh y d y
$$

decreases and thus $K_{0}(s f(u))$ increases. By the monotone convergence theorem

$$
g(y)=\frac{2}{\pi} \int_{0}^{\infty} d s \cosh (s / 2 y) K_{0}(s) .
$$

Since $K_{0}(s) \sim e^{-s} s^{-1 / 2}$ at infinity, and $\int_{0}^{\infty} K_{0}(s) d s=\frac{\pi}{2}$, all the results about $g$ are evident.

(iii) Since $K_{0}(s) \geqq d e^{-s}(s+1)^{-1 / 2},($ A.2.7) implies that for $\ell$ large

$$
(2 \ell+1)^{-1} \hat{D}_{\ell}^{2}\left(\frac{1}{2}(2 \ell+1)-\sqrt{2 \ell+1}\right)
$$

$$
\geqq c \int_{(2 \ell+1)^{-1 / 3}}^{\infty} d t\{\exp [(2 \ell+1)(t-\sinh t)+\sqrt{2 \ell+1} \sinh t]\}[(2 \ell+1) \sinh t]^{-1 / 2} .
$$

In the region of integration $(2 \ell+1)^{-1 / 3}<t<2(2 \ell+1)^{-1 / 3},(2 \ell+1)(t-\sinh t)$ is bounded, and the factors from $[(2 \ell+1) \sinh t]^{-1 / 2}$ and $\left(\int d t\right)$ only go to zero as an inverse power of $\ell$ while $\exp ((\sqrt{2 \ell+1}) \sinh t)$ blows up like $\exp (2 \ell+1)^{1 / 6}$ 
Lemma A.2.4. Define $x_{\ell}, w_{\ell}$ by $\hat{\delta}_{\ell}\left(x_{\ell}\right)=\pi / 12$ and $\hat{D}_{\ell}^{2}\left(w_{\ell}\right)=2$. Then

$$
\sup _{\ell}\left\{(2 \ell+1)^{-1} \int_{x_{\ell}}^{w_{\ell}} \hat{D}_{\ell}^{2}(x) d x\right\}=B<\infty .
$$

Proof. By (A.2.6) and (i) of the last lemma

$$
\hat{\delta}_{\ell}(x) \leqq x \hat{D}_{\ell}^{-2}(x)
$$

and thus, by (iii) of the last lemma

$$
\lim _{\ell}\left[\hat{\delta}_{\ell}\left(\frac{1}{2}(2 \ell+1)-\sqrt{2 \ell+1}\right)\right]=0 .
$$

It follows that for $\ell$ large

$$
x_{\ell} \geqq \frac{1}{2}(2 \ell+1)-\sqrt{2 \ell+1}
$$

and that

$$
\begin{aligned}
\frac{\pi}{12} & =\hat{\delta}_{\ell}\left(x_{\ell}\right)-\lim _{\ell} \hat{\delta}_{\ell}\left(\frac{1}{2}(2 \ell+1)-\sqrt{2 \ell+1}\right) \\
& \left.\leqq \lim \left[\left\{x_{\ell}-\left[\frac{1}{2}(2 \ell+1)-\sqrt{2 \ell+1}\right)\right]\right\} \hat{D}_{\ell}^{-2}\left(x_{\ell}\right)\right]
\end{aligned}
$$

so that

$$
\hat{D}_{\ell}^{2}\left(x_{\ell}\right) \leqq C \sqrt{2 \ell+1}
$$

since either $x_{\ell} \leqq \frac{1}{2}(2 \ell+1)+\sqrt{2 \ell+1}$ in which case (A.2.17) follows from (A.2.16) or $x_{\ell} \geqq \frac{1}{2}(2 \ell+1)+\sqrt{2 \ell+1}$ in which case (A.2.17) follows from (A.2.10) and (A.2.11). Next, note that

$$
\int_{x_{\ell}}^{w_{\ell}} \hat{D}_{\ell}^{2}(x) d x \leqq a+b
$$

where

$$
a=\int_{x_{\ell}}^{1 / 2(2 \ell+1)} \hat{D}_{\ell}^{2}(x) d x
$$

and

$$
b=\int_{1 / 2(2 \ell+1)}^{w_{\ell}} \hat{D}_{\ell}^{2}(x) d x
$$

and $a$ is only present if $x_{\ell}<\frac{1}{2}(2 \ell+1)$. Clearly since $D$ is monotone,

$$
a \leqq\left[-x_{\ell}+\frac{1}{2}(2 \ell+1)\right] \hat{D}_{\ell}\left(x_{\ell}\right)^{2}=O((2 \ell+1))
$$

by (A.2.15) and (A.2.17) and,

$$
(2 \ell+1)^{-1} b_{\ell} \rightarrow \int_{1 / 2}^{w_{\infty}} g(y) d y<\infty
$$

by (A.2.9) where $\tilde{w}_{\infty}$ is determined by $g\left(\tilde{w}_{\infty}\right)=2$. 
Lemma A.2.5. If $V$ obeys (A.2.1) with $\alpha>1$, then

$$
-\delta_{\ell}(g) \leqq \frac{\pi}{12}+2(B+1)(g A)(1+\ell)^{-(\alpha-1)}
$$

for all $\ell \geqq \ell_{0}$ for a $g$-independent $\ell_{0}$. Moreover, if $\ell \geqq \ell_{0}$ and $g$ are such that $-\delta_{\ell}(g)$ $\leqq(\pi / 6)$, then

$$
-\delta_{\ell}(g) \geqq C g(1+\ell)^{-(\alpha-1)} .
$$

Proof. By (A.2.3)-(A.2.5)

$$
-\delta_{\ell}(g) \leqq \frac{\pi}{12}+g \int_{x_{\ell}}^{\infty} V(x) \hat{D}_{\ell}^{2}(x) d x
$$

For $\ell$ large, the integral from $x_{\ell}$ to $w_{\ell}$ is bounded by $g V\left(x_{\ell}\right) \int_{x_{\ell}}^{w_{\ell}} D_{\ell}^{2}(x) d x$ $\leqq g A(1+\ell)^{-\alpha} 2 B(\ell+1)$ and again for $\ell$ large,

$$
g \int_{w}^{\infty} V(x) \hat{D}_{\ell}^{2}(x) d x \leqq 2 g \int_{w}^{\infty} V(x) \leqq(g A) 2(1+\ell)^{-(\alpha-1)}
$$

This proves (A.2.18). To prove (A.2.19), we note that if $-\delta_{\ell}(g) \leqq \pi / 6$, then $-d_{\ell}(g, r)$ $\leqq \pi / 6$ for all $r$, so $\sin ^{2}\left(\hat{\delta}_{\ell}+d_{\ell}\right) \geqq \sin ^{2}(\pi / 6)$ for all $r$ with $\pi / 3 \leqq \hat{\delta}_{\ell} \leqq 2 \pi / 3, \bmod 2 \pi$. Since $\frac{1}{2} \leqq \hat{\delta}_{\ell}^{\prime} \leqq 1$ for $r \geqq w_{\ell}$, we have contributions from enough intervals for

$$
\int_{w_{\ell}}^{\infty} V(r) \sin ^{2}\left(\hat{\delta}_{\ell}+d_{\ell}\right) d r \geqq(\text { const }) \int_{w_{t}}^{\infty} V(r) d r
$$

whence (A.2.19) follows.

Proof of Theorem A.2.2. By (A.2.18), $-\delta_{\ell} \leqq \pi / 6$ eventually so for $\ell \geqq \ell_{0}, \sin ^{2} \delta_{\ell}$ $\geqq \delta_{\ell}^{2}[(6 / \pi) \sin (\pi / 6)]^{2}$ and thus by (A.2.19),

$$
\sum_{\ell_{0}}^{\infty}(2 \ell+1) \sin ^{2} \delta_{\ell} \geqq c \sum_{\ell_{0}}^{\infty}(2 \ell+1)(1+\ell)^{-2(\alpha-1)}
$$

diverges.

Proof of Theorem A.2.1. As in the last proof, we pick $\ell_{0}(g)$ so that

$$
\sigma_{\text {tot }}(g) \geqq c g^{2} \sum_{\ell_{0}(g)}^{\infty}(2 \ell+1)(1+\ell)^{-2(\alpha-1)} .
$$

By (A.2.18), we must take $\ell_{0}(g) \sim d g^{1 /(\alpha-1)}$ for $d$ small. Thus

$$
\begin{aligned}
\sigma_{\text {tot }} & \geqq C^{\prime} g^{2}\left[\ell_{0}(g)\right]^{-2 \alpha+4} \\
& \geqq d^{\prime} g^{2} g^{(4-2 \alpha) /(\alpha-1)}=d^{\prime} g^{2 /(\alpha-1)} .
\end{aligned}
$$




\section{Appendix 3. Bounds on Cross Sections by the Birman-Krein Theory}

The total cross-section averaged over initial angles is just the square of the HilbertSchmidt norm of a constant energy fiber $(S(\lambda)-1)$ of $S-1$ with $S$ the $S$-operator. But

$$
\begin{aligned}
\|(S(\lambda)-1)\|_{2}^{2} & =\operatorname{Tr}\left((S(\lambda)-1)^{*}(S(\lambda)-1)\right) \\
& =2 \operatorname{Re}(\operatorname{Tr}(1-S(\lambda))) \\
& \leqq 2\|S(\lambda)-1\|_{1}
\end{aligned}
$$

with $\|\cdot\|_{1}=$ trace class norm. Thus, the celebrated results of Birman and Krein [8] bounding $\|S(\lambda)-1\|_{1}$ give control on the total cross-section. These are only applicable for potentials with more-or-less $r^{-v-\varepsilon}$ falloff at infinity. Our goal in this appendix is to show how to recover the correct large coupling constant behavior for $V$ 's with $|x|^{-\alpha}$ falloff $(\alpha>v)$ using the Birman-Krein theory. Other trace class estimates have been obtained by Davies [15].

Given operators $A, B$ with complete scattering, one can form the $S$-operator $S(A, B)=\Omega^{-}(A, B)^{*} \Omega^{+}(A, B)$. Since $S(A, B)$ commutes with $B$, it can be realized naturally in terms of the integral decomposition $B=\int^{\otimes} \lambda 1 d \mu(\lambda)$ describing $B$. We denote the fibers in this decomposition by $S(A, B ; \lambda): \mathscr{H}_{\lambda}(B) \rightarrow \mathscr{H}_{\lambda}(B)$. The basic result we will apply is that of Birman and Krein [8] combined with the invariance principle:

Theorem A.3.1. If $e^{-A}-e^{-B} \in \mathscr{I}_{1}$, then $\Omega^{ \pm}(A, B)$ exist and are complete, $S(A, B ; \lambda)-1$ is trace class for almost every $\lambda$ and

$$
\int\|S(A, B ; \lambda)-1\|_{1} e^{-\lambda} d \lambda \leqq 2 \pi\left\|e^{-A}-e^{-B}\right\|_{1} .
$$

We will also need the following result abstracted from [8]:

Proposition A.3.2. If both $e^{-A}-e^{-B}$ and $e^{-B}-e^{-C_{\varepsilon}} \mathscr{I}_{1}$, then for almost all $\lambda$

$$
\|S(A, C ; \lambda)-1\|_{1} \leqq\|S(A, B ; \hat{\lambda})-1\|_{1}+\|S(B, C ; \lambda)-1\|_{1} .
$$

Remark. $S(A, C ; \lambda)$ and $S(A, B ; \lambda)$ act on "different" spaces, namely the fibers of the direct integral decomposition for $C$ and $B$ respectively. Of course, the fibers of $\Omega^{+}(B, C)\left[\right.$ or $\left.\Omega^{-}(B, C)\right]$ set up a natural isometry between the two spaces.

We will use these results and the method of Deift and Simon [16] to estimate $\|S(A, B ; \lambda)-1\|_{1}$ for $A=-\Delta+V, B=-\Delta$. For other results on this case by these methods, see $[13,38,43]$. For simplicity, we suppose throughout that $V \geqq 0$. We will exploit the space $\ell^{1}\left(L^{2}\right)$ of Birman and Solomjak [9]:

Definition. Let $\Delta_{\alpha}, \alpha \in Z^{v}$ be the unit cube in $R^{v}$, centered about $\alpha . \ell^{1}\left(L^{2}\right)$ is the set of functions $V$ on $R^{v}$ with

Here we will prove:

$$
\|V\|_{2 ; 1} \equiv \sum_{\alpha}\left[\int_{A_{\alpha}}|V(x)|^{2} d^{v} x\right]^{1 / 2}<\infty .
$$

Theorem A.3.3. If $V \geqq 0, V \in \ell^{1}\left(L^{2}\right)$ and $S(\lambda)$ are the fibers of $S(-\Delta+V,-\Delta)$, then

$$
\int_{0}^{\infty}\|1-S(\lambda)\|_{1} e^{-\lambda} d \lambda \leqq C\|V\|_{2 ; 1} .
$$


Theorem A.3.4. Under the hypotheses of Theorem A.3.3, for any $R \geqq 1$ :

$$
\int_{0}^{\infty}\|1-S(\lambda)\|_{1} e^{-\lambda} d \lambda \leqq C\left[R^{v-1}+\left\|V \chi_{R}\right\|_{2 ; 1}\right]
$$

with $\chi_{R}$ the characteristic function of $\{x|| x \mid>R\}$.

Remarks. 1 . The parameter $\lambda$ here is the total energy to be compared with the parameter $k \sim \lambda^{1 / 2}$ used in the bulk of the paper. Thus, when there is a zero energy resonance, $\|S(\lambda)-1\|_{1} \sim \lambda^{-1 / 2}$ at zero, a divergence consistent with these theorems.

2. If $0 \leqq V(x)<(1+|x|)^{-\alpha}$ with $\alpha>v$, then $\left\|V \chi_{R}\right\|_{2 ; 1} \leqq c R^{v-\alpha}$ so if $V$ is replaced by $g V$, by minimizing $R^{v-1}+g R^{v-\alpha}$ we discover a $g^{v-1 / \alpha-1}$ bound consistent with our results in the bulk of the paper.

3. Dirichlet decoupling as used by Deift and Simon [16] yields the $R^{v-1}$ behavior we find in Theorem A.3.4. The alternative method to [16] introduced by Combescure and Ginibre [13] yields an $R^{v}$ behavior in that place and hence worse coupling constant bounds.

We use the following notation: $-\Delta_{\Gamma}$ is the Dirichlet Laplacian with vanishing boundary conditions on $\Gamma$; and $H(\Gamma ; V) \equiv-\Delta_{\Gamma}+V$. We begin with the following estimate which is essentially Corollary $25.10 \mathrm{~B}$ of [47]:

Lemma A.3.5. Let $f \in \ell_{1}\left(L^{2}\right), V \geqq 0, t \geqq 1 / 2, \Gamma$ arbitrary. Then $f e^{-t H(\Gamma ; V)} \in \mathscr{I}_{1}$ and

$$
\left\|f e^{-t H(r ; V)}\right\|_{1} \leqq c\|f\|_{2 ; 1} .
$$

Proof. Suppose first that $f$ has support in the unit cube centered at 0 . Write

$$
f e^{-t H(\Gamma ; V)}=A B
$$

with

$$
\begin{aligned}
& A=f e^{-t H(\Gamma ; V) / 2}\left(1+|x|^{2}\right)^{v} \\
& B=\left(1+|x|^{2}\right)^{-v} e^{-t H(\Gamma ; V) / 2} .
\end{aligned}
$$

Since $e^{-s H(\Gamma ; V)} \leq e^{-s H_{0}}$ (where $C \leq D$ means the integral kernel for $D$ dominates that for $C$ ), we see that $A$ and $B$ are Hilbert-Schmidt with $\|A\|_{2} \leqq c\|f\|_{2}$. Thus, by translation covariance, for any $f \in L_{\mathrm{lcc}}^{2}$ :

$$
\left\|f \chi_{\alpha} e^{-t H(\Gamma ; V)}\right\|_{1} \leqq c\left\|f \chi_{\alpha}\right\|_{2} .
$$

(A.3.1) follows from summing over $\alpha$.

Lemma A.3.6. Let $V, W \geqq 0$. Let $H=-\Delta_{\Gamma}+V ; H^{\prime}=H+W$. Then

$$
\left\|e^{-H}-e^{-H^{\prime}}\right\|_{1} \leqq c\|W\|_{2 ; 1} \text {. }
$$

Proof.

$$
\begin{aligned}
\left\|e^{-H}-e^{-H^{\prime}}\right\|_{1} & =\left\|\int_{0}^{1} e^{-\theta H} W e^{-(1-\theta) H^{\prime}} d \theta\right\|_{1} \\
& \leqq \int_{0}^{1 / 2}\left\|W e^{-(1-\theta) H^{\prime}}\right\|_{1} d \theta+\int_{1 / 2}^{1}\left\|e^{-\theta H} W\right\|_{1} d \theta
\end{aligned}
$$

so that (A.3.2) follows from (A.3.1). 
Proof of Theorem A.3.3. By (A.3.2), with $V=0, \Gamma=\emptyset$ and $W$ changed to $V$,

$$
\left\|e^{-(-\Delta+V)}-e^{+\Delta}\right\|_{1} \leqq C\|V\|_{2 ; 1} \text {. }
$$

Theorem A.3.1 now concludes the proof.

Lemma A.3.7. Let $\Gamma^{\prime}$ lie in a unit cube. Then

$$
\left\|e^{-t H\left(\Gamma \cup \Gamma^{\prime} ; V\right)}-e^{-t H(\Gamma ; V)}\right\|_{1} \leqq c
$$

with $c$ only depending on $t$.

Proof. By translation invariance we may assume that $\Gamma^{\prime}$ lies in the unit cube at the origin. Let $A=e^{-t H\left(\Gamma \cup \Gamma^{\prime} ; V\right) / 2}, B=e^{-t H(\Gamma ; V) / 2}$. Then $\left(1+x^{2}\right)^{-v} A$ and $B\left(1+x^{2}\right)^{-v}$ are Hilbert-Schmidt since $e^{-s H(G ; V)} \leq e^{-s H_{0}}$. Thus, writing

$$
A^{2}-B^{2}=\left[(A-B)\left(1+x^{2}\right)^{v}\right]\left(1+x^{2}\right)^{-v} A+B\left(1+x^{2}\right)^{-v}\left[\left(1+x^{2}\right)^{v}(A-B)\right]
$$

we see that it suffices that $\left(1+x^{2}\right)^{v}(A-B)$ is Hilbert-Schmidt. But $B-A \leq B_{0}-A_{0}$ where $A_{0}, B_{0}$ are the operators with $\Gamma=\emptyset, V=0$. By standard estimates $[16,47]$ :

$$
\left(B_{0}-A_{0}\right)(x, y) \leqq c e^{-d[|x|+|y|]} .
$$

Definition. For any set $S \subset \mathbb{R}^{v}$, let $n(S)=$ the number of unit cubes, $C$, centered at integral lattice points with $C \cap S \neq \emptyset$.

By an easy induction, Lemma A.3.7 immediately implies

Lemma A.3.8. For any $\Gamma^{\prime}, \Gamma, V \geqq 0$ :

$$
\left\|e^{-t H\left(\Gamma \cup \Gamma^{\prime} ; V\right)}-e^{-t H(\Gamma ; V)}\right\|_{1} \leqq C n\left(\Gamma^{\prime}\right) .
$$

Proof of Theorem A.3.4. Let $H=-\Delta+V, H_{0}=-\Delta, H^{\prime}=-\Delta_{\Gamma}+V, H_{0}^{\prime}=-\Delta_{\Gamma}$ with $\Gamma$ the sphere of radius $R$. Following [16], we take scattering $\left(H_{0}, H\right)$ in three steps: $\left(H_{0}, H_{0}^{\prime}\right),\left(H_{0}^{\prime}, H^{\prime}\right),\left(H^{\prime}, H\right)$. By Proposition A.3.2 we need only prove a bound on $\int_{0}^{\infty}\|S(A, C ; \lambda)-1\|_{1} e^{-\lambda} d \lambda$ for each pair $(A, C)$. By Lemma A.3.8 and Theorem A.3.1, the $\left(H_{0}, H_{0}^{\prime}\right)$ and $\left(H, H^{\prime}\right)$ integrals are bounded by $c R^{v-1}$. Because of Dirichlet decoupling the scattering for $\left(H_{0}^{\prime}, H^{\prime}\right)$ is the same as that for $\left(H_{0}^{\prime}, H_{0}^{\prime}+V \chi_{R}\right)$ and therefore, by Lemma A.3.6 and Theorem A.3.1, the corresponding weighted integral of $\|S-1\|_{1}$ is bounded by $c\left\|V \chi_{R}\right\|_{2 ; 1}$.

\section{Appendix 4. Kato's Monotonicity Theorem and Scattering From Potentials with Small Support}

Our goal here is to prove:

Theorem A.4.1. Let $v \geqq 3$. Fix $k>0$ and let $\sigma_{R}(k)$ denote the total cross section for a hard core potential of radius $R$. Let $V$ be any $\left(L^{v / 2+\varepsilon}\right)$ potential supported in $\{x|| x \mid<R\}$ with $0 \leqq V$. Then for all sufficiently small $R$,

$$
\sigma(k, V) \leqq \sigma_{R}(k)
$$


Remarks. 1. By scaling, taking $R$ to zero is the same as taking $k$ to zero, i.e., for any fixed $R$, (A.4.1) holds for $k$ sufficiently small, or equivalently for each $R$, (A.4.1) holds for $k R$ sufficiently small.

2. The result is true if $v=2$ where $\delta_{0}^{R} \sim[\ln (k R)]^{-1}$ but as we have not discussed this case in Appendix 1, we state the result for $v>3$.

3. The proof does not extend to $v=1$ dimension where $d_{0}^{R} \sim-\pi / 2$ and $\sigma_{R}(k)=2$ independently of $R$ and $k\left(\delta_{0}\right.$ and $\delta_{1}$ differ by $\left.\pi / 2\right)$. Presumably, the result is even false in this case.

4. For central potentials, Kato's theorem used below can be replaced by wellknown results (see e.g., Calogero [10]), i.e., by (A.2.5).

5. As explained in Appendix $1, \sigma_{R}(k)=O\left(R^{2 v-4}\right)$ for $R$ small.

Proof. In [30], Kato proves a monotonicity result, which shows that the total phase shift $\theta(V)$ can be defined for $V \geqq 0$ so that (i) $\theta(V) \leqq 0$ for $V \geqq 0$; (ii) $\theta(V) \leqq \theta(W)$ if $V \geqq W \geqq 0$; (iii) $\theta(\lambda W) \rightarrow 0$ as $\lambda \downarrow 0$. (iv) If $\theta_{R}$ is the sum of the phase shifts of the hard core problem of radius $R,-\theta(\lambda V) \leqq-\tilde{\theta}_{R} ;$ (v) the individual eigenvalues $\theta_{n}$ of $\ln S$ are monotone in the region where all $\theta_{n}$ stay away from 0 and if $0<-\theta_{n}<\pi, \theta=\sum_{n} \theta_{n}$.

From these facts, we conclude that if $\tilde{\theta}_{R}<\pi / 2$, all eigenvalues $\theta_{n}$ lie in $(0, \pi / 2)$ and since $\sin ^{2} \theta$ is monotone on $(0, \pi / 2), \sigma(k, V) \leqq \sigma_{R}(k)$. Thus the result follows from the observation that $\tilde{\theta}_{R}<\pi / 2$ for small $R$ by the discussion in Appendix 1.

\section{References}

1. Agmon, S.: Utah lectures (in preparation) (1978)

2. Alonso y Coria, A.: Princeton University Thesis, 1978

3. Alsholm, P., Schmidt, G.: Arch. Rat. Mech. Anal. 40, 281 (1971)

4. Amrein, W., Pearson, D.: J. Phys. A12, 1469 (1979)

5. Amrein, W., Pearson, D., Sinha, K.: Il Nuovo Cimento 52A, 115 (1979)

6. Babikov, V.V.: The Method of Phase Functions in Quantum Mechanics (in Russian), Nauka, Moscow 1968; Sov. Phys. Uspekhi 92, 271 (1967)

7. Birman, M., Borzov, V.: Probs. Math. Phys. 5, 24 (1971)

8. Birman, M., Krein, M.: Soviet Math. Dokl. 3, 740 (1962)

9. Birman, M., Solomjak, M.: Vest. LSU 24 (1969)

10. Calogero, F.: The Variable Phase Approach to Scattering. New York: Academic Press 1967

11. Combes, J.M. : In preparation

12. Combes, J.M., Tip, A. : In preparation

13. Combescure, M., Ginibre, J.: J. Func. Anal. 29, 54 (1978)

14. Cwickel, M.: Ann. Math. 106, 93 (1977)

15. Davies, E.B.: Oxford preprint

16. Deift, P., Simon, B.: J. Func. Anal. 23, 218 (1976)

17. Dollard, J.: Commun. Math. Phys. 12, 193 (1969)

18. Enss, V.: Commun. Math. Phys. 52, 233 (1977)

19. Enss, V.: Commun. Math. Phys. 61, 285 (1978)

20. Enss, V., Simon, B. : Phys. Rev. Lett. 44, 319 (1980) Erratum: Phys. Rev. Lett. 44, 764 (1980)

21. Farıs, W.: Rocky Mt. J. Math. 1, 637 (1971)

22. Friedman, C.: J. Func. Anal. 10, 346 (1972)

23. Flügge, S. : Practical Quantum Mechanics. Berlin. Heidelberg, New York: Springer 1974 
24. Grossman, A., Hoegh-Krohn, R., Mebkhout, M.: J. Math. Phys. (to appear)

25. Hagedorn, G.: Commun. Math. Phys. 71, 77 (1980)

26. Hörmander, L.: Math. Zeit. 146, 69 (1976)

27. Ikebe, T.: Arch. Rat. Mech. Anal. 5, 1 (1960)

28. Jauch, J., Lavine, R., Newton, R.: Helv. Phys. Acta 45, 325 (1972)

29. Jensen, A.: Duke Math. J. (to appear)

30. Kato, T.: Hadronic J. 1, 134 (1978)

31. Klaus, M., Simon, B.: Ann. Phys. (to appear)

32. Klaus, M., Simon, B.: Commun. Math. Phys. (to appear)

33. Kupsch, J., Sandhas, W.: Commun. Math. Phys. 2, 147 (1966)

34. Kuroda, S. : J. Fac. Sci. Tokyo 17, 315 (1970)

35. Lieb, E.: Bull. AMS 82, 751 (1976) and Proc. AMS Hawaii Symp. 1979 (to appear)

36. Martin, A.: Helv. Phys. Acta 45, 140 (1972)

37. Martin, A.: Commun. Math. Phys. 69, 89 (1979)

38. Pearson, D.: Helv. Phys. Acta 48, 249 (1976)

39. Peierls, R.: Surprises in Theoretical Physics. Princeton : Princeton University Press 1979

40. Reed, M., Simon, B.: Methods of Modern Mathematical Physics. III. Scattering Theory. New York: Academic Press 1979

41. Reed, M., Simon, B.: Methods of Modern Mathematical Physics. IV. Analysis of Operators. New York: Academic Press 1978

42. Rosenbljum, C.V.: Soviet Math. Dokl. 13, 245 (1972)

43. Semenov, Yu.: Lett. Math. Phys. 1, 457 (1977)

44. Simon, B.: Helv. Phys. Acta 43, 607 (1970)

45. Simon, B.: Commun. Math. Phys. 55, 259 (1977)

46. Simon, B.: Commun. Math. Phys. 58, 205 (1978)

47. Simon, B.: Functional Integration and Quantum Physics. New York: Academic Press 1979

48. Tamura, H.: Proc. Japan Acad. 50, 19 (1974)

49. Thoe, D.: Rat. Mech. Anal. 26, 335 (1967)

50. Thomas, L.: J. Math. Phys. 20, 1848 (1979)

51. Yajima, K.: Commun. Math. Phys. 69, 101 (1979)

52. Zemach, C., Klein, A. : Nuovo Cimento 10, 1078 (1958)

Communicated by J. Ginibre

Received April 14, 1980 
